\title{
Article \\ The Network Topology Metrics Contributing to Local-Area Frequency Stability in Power System Networks
}

\author{
Warren J. Farmer* (D) and Arnold J. Rix (D) \\ Departmen Electrical and Electronic Engineering, Stellenbosch University, Stellenbosch 7600, South Africa; \\ rix@sun.ac.za \\ * Correspondence: 17647533@sun.ac.za
}

Citation: Farmer, W.J.; Rix, A.J. The Network Topology Metrics Contributing to Local-Area Frequency Stability in Power System Networks. Energies 2021, 14, 4643. https://doi.org/10.3390/en14154643

Academic Editor: Ali Mehrizi-Sani

Received: 17 June 2021

Accepted: 26 July 2021

Published: 30 July 2021

Publisher's Note: MDPI stays neutral with regard to jurisdictional claims in published maps and institutional affiliations.

Copyright: (c) 2021 by the authors. Licensee MDPI, Basel, Switzerland. This article is an open access article distributed under the terms and conditions of the Creative Commons Attribution (CC BY) license (https:// creativecommons.org/licenses/by/ $4.0 /)$.

\begin{abstract}
The power system network topology influences the system frequency response to power imbalance disturbances. Here, the objective is to find the network metric(s) contributing to frequency transient stability. The graph Laplacians of six 4-node network topologies are analysed using Spectral Graph Theory. For homogeneous network connections, we show that the node degree measure indicates node robustness. Based on these analytical results, the investigation expands to a 10-node network topology consisting of two clusters, which provide further insight into the spectral results. The research then involves a simulation of a power imbalance disturbance on three 20-node networks with different topologies based on node degree, where we link the node degree measure to imbalance disturbance propagation through Wave Theory. The results provide an intuitive understanding of the impact of network topology on power system frequency stability. The analytical and simulation results indicate that a node's sensitivity to disturbances is partially due to its node degree, reactance from disturbance location, and the link it has to other higher degree nodes (hierarchical position in network topology). Testing of the analytical and simulation results takes place on the nonhomogeneous IEEE-14 bus and IEEE-39 bus networks. These results provide insights into optimal inertia placement to improve the frequency robustness of low-inertia power systems. The network topology, considering node degrees, influences the speed at which the disturbance impact propagates from the disturbance location and how fast-standing waves form. The topology thus contributes to how fast the energy in a disturbance dissipates to zero.
\end{abstract}

Keywords: power system; disturbance; network topology; frequency stability; graph laplacian

\section{Introduction}

Frequency stability is critical for power system operation [1]. The inertia of synchronous generators provides the power system with frequency transient stability [2-4]. However, the large-scale transition from fossil fuel power stations (which primarily use synchronous generators) to variable renewable energy source (RES) power plants-like wind and solar photovoltaic (PV) - means there is a shift from synchronous generators to inverterbased generation [5-7]. The higher dispatch priority for RES generation above conventional fossil fuel generation results in reduced and time-varying system inertia. The inertia quantity's time-varying component is due to the residual load becoming more intermittent as a result of the stochasticity and variability introduced by large-scale inverter-based RES generation $[8,9]$. The inertia of synchronous generators is essential for system frequency stability during a generation/load imbalance disturbance event and prior to governor action. Thus, a consequence of large-scale RES integration is the decline in synchronous generator inertia, which degrades the power system's frequency transient stability [10-13]. A high rate of change of frequency $(\mathrm{RoCoF})$ can lead to the activation of distance protection, network splitting, and in the worst case, a system-wide blackout [14-16]. To accommodate large-scale inertia-less generation integration necessitates deeper insight into the power system dynamics to improve and ensure system frequency transient stability. 
The ongoing integration of distributed RES increases the complexity of the network structure. It becomes thus necessary to perform the $N-1$ contingency and stability assessments over larger and more complex networks [17]. The increase in nonlinear differential equations increases the computational load to complete $\mathrm{N}-1$ assessments.

For power system networks with long transmission lines, i.e., high impedance, the frequency dynamics are less coherent between buses. The frequency transient response and robustness are not uniform across a synchronised network. Therefore, the location of a network disturbance is of importance in the assessment of frequency stability.

A system norm is helpful to assess a system's stability performance to a specific disturbance vector. The system norm indicates the system's sensitivity to a given disturbance input [18]. For assessing, comparing, or optimising a network's stability, it is best to use a system norm such as the $H_{2}$ or the $H_{\infty}$ norm [18], which indicates the amplification in system energy due to the disturbance input. Despite the advantages of the norms, an investigation by M. Pirani et al. [18] found that the $H_{2}$ and $H_{\infty}$ are, in general, not consistent and produce contradictory results in some instances.

There is thus a need for improved modelling, analysing methods, and insight to understand robust network design and analysis $[6,19]$. The current theoretical analysis, underlying assumptions and models need to be questioned in the context of low and variable inertia [9] to improve and update our understanding and representation of the power system. The transition from traditional and centralised to variable and spatially distributed generation (inverter-based RES) necessitates new methods/approaches for faster stability assessments.

A transmission network is a medium for disturbance propagation. The network topology, therefore, has a role in frequency transient stability. S. Bhela et al. [20] points out that the impact of network topology on frequency stability is still unknown. E. Tegling et al. [21], and M. Pirani et al. [18], indicate for low-inertia power systems that the ability to maintain frequency transient stability depends on network topology, nodal dynamics, and coupling strength.

In network structure and topology analysis, it might be valuable to identify the most influential nodes in the network, such as the work by G. Murić et al. [22] in epidemic spreading dynamics. There are various definitions for node importance depending on the context and spreading process. Node importance or criticality is typically in terms of centrality measures. G. Murić et al. [22] view node importance from the perspective of epidemic dynamics where, for example, the expected force metric quantifies the spreading power of a node. T. Coletta et al. [7] conclude that the resistive closeness centrality is relevant to identify the crucial nodes. M. Tyloo and T. Coletta et al. [23], furthermore, state that a network's synchronisation depends on a family of topological indices, which they encapsulate in the Kirchhoff index, which serve as a network metric and not as a node topological metric.

U. Markovic et al. [19] found that reducing network connectivity increases the dependence on synchronous generation for system frequency stability. The same authors state that network connectivity contributes to the maximum allowable inverter penetration in a power system network. The authors, furthermore, suggest research on the impact of unit placement on transient dynamics and stability considering high inverter penetration.

J. S. Thorp et al. [24] postulate that the location and trajectory of the eigenvalues near the instability region could provide important information about the effect of variation in system parameters on stability or where the highest potential for problems in a network is.

Through Spectral Graph Theory, L. Pagnier and Ph. Jacquod [25] have shown that the location of a disturbance in a network contributes significantly to the network's transient response. The authors found that the eigenvector belonging to the smallest non-zero eigenvalue of the network Laplacian indicates the robustness of the node mapped to the eigenvector component. The smaller the eigenvector component is, the more robust the corresponding node. The topological metrics defining this local-area robustness in a power system network is, however, still unknown. 
H. Ronellenfitsch et al. [26] proposed and investigated the concept of noise-cancelling networks. The objective was to minimise the impact of noise input using the network topology. The results of $\mathrm{H}$. Ronellenfitsch et al. points to sparse network architectures with a sense of hierarchical structures, which resemble the vascular in plants and animals. This finding aligns with the results from S. Bhela et al. [20], who found that the tree topology is the optimal design to reduce the amplitude of the generator rotor swings. $\mathrm{S}$. Bhela et al. [20] found that desynchronisation through the network topology is valuable for minimising the impact of noise input (fluctuating power imbalance disturbances). There are, however, no insights into how the topology operates/functions to minimise the disturbance impact globally.

In summary, power systems transition to less stable and more complex versions. Ensuring a smooth transition process requires insights into network topology dynamics and optimal unit placement. There is thus a need for robust network design to accommodate the transition in generation type, i.e., from synchronous generators to inverter-based RES. A valuable component for a frequency-stable network topology design is the identification of critical nodes or buses. Identification and obtaining insight into the node topology structure for a given power system network is the premise of this research.

Instead of searching for a network topology metric, like the Kirchhoff index, this research focuses on the individual node/bus topological metric to identify frequency-stable areas within a network. Unlike G. Murić et al. [22], we are not interested in spreading power or most influential but in robustness. This research expands on the work in the literature. The work in this paper provides insight into how network topology impacts frequency stability by understanding the spectrum of the network Laplacian based on the spectral analysis of [25]. The focus is on the nodal topology measure determining relative robustness in a network.

The contributions of this research to the literature are as follows: Identify the topological metrics that determine the magnitude of the eigenvector component (from the graph Laplacian) for the corresponding network bus. The second part presents how a localised disturbance propagates in a wave-like nature, impacting the rest of the power system network. We link the node degree measure to imbalance disturbance propagation through Wave Theory. The propagation velocity result aligns with the results of J. S. Thorp et al. [24], and M. Parashar et al. [27]. The two contributions show how the node topology measure responsible for distinguishing different stabilities/sensitivities between nodes dissolves the impact of a power imbalance disturbance on a network. The insights obtained are valuable for the identification of stable and vulnerable areas in a power system network, as well as for robust network design. However, it is necessary to recognise that the intention of the topology measure in this context is not to compare different networks but nodes within a given network.

The rest of the paper outline is as follows: Section 2 presents the analytical graph spectral analysis. Section 3 focuses on disturbance propagation based on the results of Section 2. Section 4 evaluates the analytical findings using the IEEE-14 bus and IEEE-39 bus systems. Section 5 summarises and discusses the findings. Finally, Section 6 presents the conclusion of this work.

\section{Network Topology Analysis}

The intention is to find the network topology metric(s), which largely determines the component sizes of the eigenvectors of the network Laplacian, to understand and classify a node's relative stability in a network. In this section, we analyse six different topologies of 4-node graphs/networks using Spectral Graph Theory. The objective is to find the network topology metric(s), which determines the magnitude of the eigenvector elements (corresponding to the smallest non-zero eigenvalue). Figure 1 presents the six different topologies. 


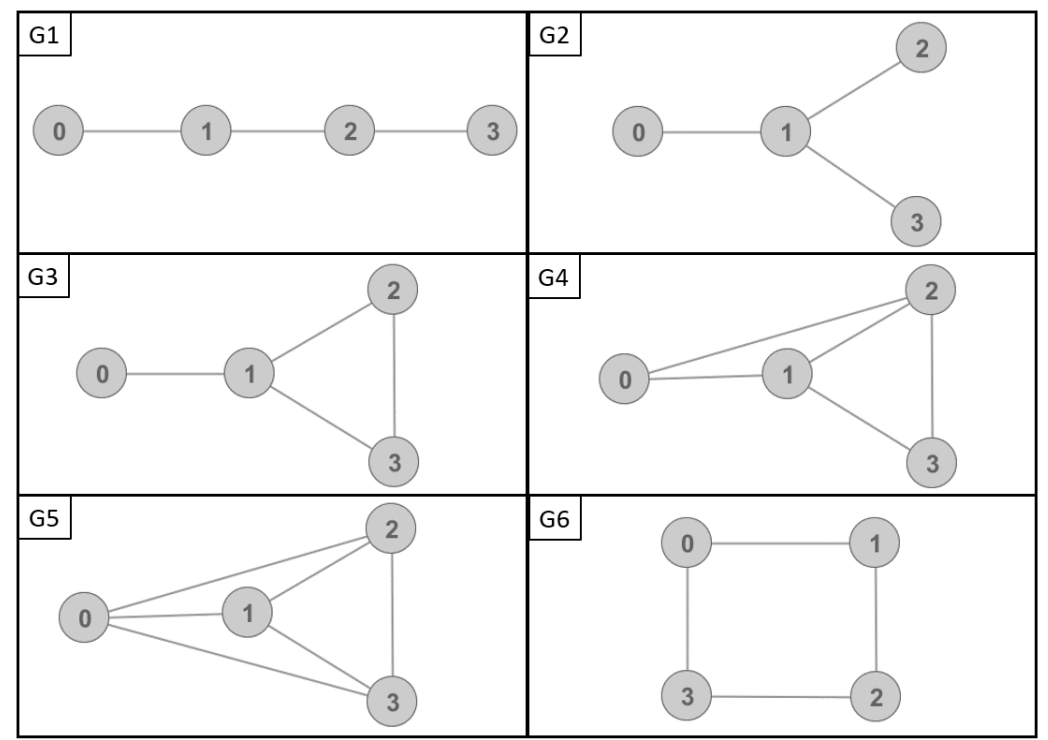

Figure 1. Six topologies of a 4-node network.

The transmission line susceptance $(b)$ is considered, for analytical simplicity, to be uniform through the networks. A graph size of four nodes is selected based on the trade-off between calculation simplicity and enough nodes to investigate various topologies. The spectrum of the networks, i.e., the eigenvalues and eigenvectors of the Laplacians' were analytically derived to obtain insight into the connection between the network topology and the Laplacian's spectrum.

For a connected graph, the Laplacian matrix is real and symmetric. The Laplacian has a complete set of orthogonal eigenvectors $\left\{\vec{u}_{1}, \ldots, \overrightarrow{u_{N}}\right\}$, with corresponding eigenvalues $\left\{\lambda_{N} \geq \ldots \geq \lambda_{2}>0\right\}$. The smallest non-zero eigenvalue $\left(\lambda_{2}\right)$ is known as the algebraic connectivity. The algebraic connectivity indicates how well a graph is connected. The larger the algebraic connectivity, the more connected the network, i.e., the network is more meshed $[17,20,21,25]$. Table 1 summarises the calculated eigenvalues (in terms of b) for graphs G1 to G6. The algebraic connectivity values are under the $\lambda_{2}$ column. The graph with the least connectivity is G1 $\left(\lambda_{2}=0.589 b\right)$ since it is a bus-topology, where the maximum node degree is two. Graph G5 is the most meshed network, and therefore, has the largest algebraic connectivity value $\left(\lambda_{2}=4 b\right)$.

Table 1. Eigenvalues of the six different topologies.

\begin{tabular}{ccccc}
\hline Eigenvalues & $\lambda_{\mathbf{1}}$ & $\lambda_{\mathbf{2}}$ & $\lambda_{\mathbf{3}}$ & $\lambda_{\mathbf{4}}$ \\
\hline G1 & 0 & $0.586 b$ & $2 b$ & $3.414 b$ \\
G2 & 0 & $b$ & $b$ & $4 b$ \\
G3 & 0 & $b$ & $3 b$ & $4 b$ \\
G4 & 0 & $2 b$ & $4 b$ & $4 b$ \\
G5 & 0 & $4 b$ & $4 b$ & $4 b$ \\
G6 & 0 & $2 b$ & $2 b$ & $4 b$ \\
\hline
\end{tabular}

Equations (1)-(6) presents the Laplacians of networks G1 to G6 with the eigenvector $\overrightarrow{u_{2}}$ corresponding to the smallest non-zero eigenvalue. For G2, G5, and G6, the algebraic connectivity has a multiplicity of more than one. Therefore, depicting the average vector $\overrightarrow{\overrightarrow{u_{2}}}$ of the corresponding vectors. The purpose is not to compare network topologies but to identify the most vulnerable areas inside a network of interest. The eigenvectors are 
normalised, thus not absolute quantities. The magnitude of the vector component indicates relative robustness to the rest of the nodes in the network. In G5 and G6, all the nodes have the same node degree and magnitudes on their respective eigenvector. Therefore, relative to each other, all the nodes in the same network have the same robustness.

For the six different topologies, the observation is that the nodes with the highest node degrees (number of directly connected neighbours) have the lowest sensitivity to disturbances, based on the eigenvector component magnitudes.

$$
\begin{aligned}
& L_{\mathrm{G} 1}:=\left[\begin{array}{cccc}
1 & -1 & 0 & 0 \\
-1 & 2 & -1 & 0 \\
0 & -1 & 2 & -1 \\
0 & 0 & -1 & 1
\end{array}\right] \quad \vec{u}_{2}=\left[\begin{array}{c}
0.653 \\
0.271 \\
-0.271 \\
-0.653
\end{array}\right] \\
& L_{\mathrm{G} 2}:=\left[\begin{array}{cccc}
1 & -1 & 0 & 0 \\
-1 & 3 & -1 & -1 \\
0 & -1 & 1 & 0 \\
0 & -1 & 0 & 1
\end{array}\right] \quad \overrightarrow{\overrightarrow{u_{2}}}=\left[\begin{array}{c}
0.333 \\
0.0 \\
0.333 \\
0.333
\end{array}\right] \\
& L_{\mathrm{G} 3}:=\left[\begin{array}{cccc}
1 & -1 & 0 & 0 \\
-1 & 3 & -1 & -1 \\
0 & -1 & 2 & -1 \\
0 & -1 & -1 & 2
\end{array}\right] \quad \overrightarrow{u_{2}}=\left[\begin{array}{c}
0.816 \\
0.0 \\
-0.408 \\
-0.408
\end{array}\right] \\
& L_{\mathrm{G} 4}:=\left[\begin{array}{cccc}
2 & -1 & -1 & 0 \\
-1 & 3 & -1 & -1 \\
-1 & -1 & 3 & -1 \\
0 & -1 & -1 & 2
\end{array}\right] \quad \overrightarrow{u_{2}}=\left[\begin{array}{c}
0.707 \\
0.0 \\
0.0 \\
-0.707
\end{array}\right] \\
& L_{\mathrm{G} 5}:=\left[\begin{array}{cccc}
3 & -1 & -1 & -1 \\
-1 & 3 & -1 & -1 \\
-1 & -1 & 3 & -1 \\
-1 & -1 & -1 & 3
\end{array}\right] \quad \overrightarrow{u_{2}}=\left[\begin{array}{l}
0.25 \\
0.25 \\
0.25 \\
0.25
\end{array}\right] \\
& L_{\mathrm{G} 6}:=\left[\begin{array}{cccc}
2 & -1 & 0 & -1 \\
-1 & 2 & -1 & 0 \\
0 & -1 & 2 & -1 \\
-1 & 0 & -1 & 2
\end{array}\right] \quad \overrightarrow{u_{2}}=\left[\begin{array}{l}
0.25 \\
0.25 \\
0.25 \\
0.25
\end{array}\right]
\end{aligned}
$$

Based on the node degree observation from the Spectral Graph Theory approach, we seek more support for the node degree and stability observation. An analytical approach, based on power flow and the Swing Equation, is taken to find an expression for the role of node degree in a node's frequency stability.

In an alternating current $(\mathrm{AC})$ network, active power flows from the larger voltage angle bus to the bus with a lower voltage angle. The power flow across a transmission line is related to the voltage phase angle difference between the ends. For a given network, its incidence matrix $\mathbf{U}$ describes the relationship between nodes and edges. Let the vector $\vec{\delta}$ depict the voltage phase angle distribution. The operation $\mathbf{U} \vec{\delta}=\Delta \vec{\delta}$ computes the voltage phase angle difference between the network buses.

Active power flow is a sinusoidal function of the phase angle difference. However, for small-angle differences, the difference approximates the sine function. Considering that power only flows between connected buses and suppose the transmission capacity between bus $i$ and $j$ is $\left(\mathbf{A}_{\mathbf{d j}}\right)_{i j}$, then the power flow through bus $i$ with any node degree is: 


$$
\begin{aligned}
P_{i} & \approx \sum_{j}\left(\mathbf{A}_{\mathbf{d j}}\right)_{i j} \cdot\left(\delta_{i}-\delta_{j}\right) \\
& \approx \delta_{i} \sum_{j}\left(\mathbf{A}_{\mathbf{d j}}\right)_{i j}-\sum_{j}\left(\mathbf{A}_{\mathbf{d j}}\right)_{i j} \delta_{j} \\
& \approx\left(\Delta \delta_{i j}+\delta_{j}\right) \operatorname{degree}\left(\operatorname{bus}_{i}\right)-\sum_{j}\left(\mathbf{A}_{\mathbf{d j}}\right)_{i j} \delta_{j} \\
& \approx \sum_{j}\left[\text { degree }\left(\text { bus }_{i}\right)-\left(\mathbf{A}_{\mathbf{d j}}\right)_{i j}\right] \delta_{j} .
\end{aligned}
$$

The above takes the following matrix and vector form:

$$
\left.\vec{P}=\left(\mathbb{D}-\mathbf{A}_{\mathbf{d j}}\right)\right) \vec{\delta}=\mathbf{U}^{\mathrm{T}} \mathbf{U} \vec{\delta}=\mathbf{L} \vec{\delta} .
$$

The discrete Laplacian is analogous to the divergence of the gradient of a function $\nabla^{2} f(\cdot)$. In the discrete graph space, $\mathbf{U}$ takes the role of $\nabla$. Thus, where $\nabla^{2}=\nabla \cdot \nabla$, the graph Laplacian is $\mathbf{L}=\mathbf{U}^{\mathrm{T}} \mathbf{U}=\mathbb{D}-\mathbf{A}_{\mathbf{d} \mathbf{j}}$.

The node degree matrix $\mathbb{D}$ is a diagonal matrix containing the degrees. Consider a power imbalance disturbance at a bus, then the higher the node degree capacity of that bus, the better the node's ability to disperse the disturbance impact energy. The adjacency matrix $\mathbf{A}_{\mathbf{d j}}$ describes the transmission capacity of the edges. The larger the edge capacity from the perturbed bus to a neighbouring bus, the easier the flow along that path.

The graph Laplacian of a given power system network describes the relationship (sensitivity) between the power flow and voltage phase angle for each network bus. A property of the Laplacian matrix is the row-sum, and the column-sum equals zero, and thus the Laplacian implies the sum of power flow is zero in a lossless network. The Laplacian matrix is also known as the Kirchhoff matrix. An expansion of $\mathbf{L} \vec{\delta}$ into its summation terms per row reveals the product of the dispersion strength and the voltage phase angle at that bus, minus the sum-of-products, of the transmission line capacity and voltage phase angles of neighbouring buses.

Consider the frequency dynamics at a node using the Swing Equation:

$$
M \frac{d}{d t} \omega+D \cdot \omega=\left(P^{(m)}+\Delta P^{(m)}\right)-\left(P^{(e)}+\Delta P^{(e)}\right) .
$$

The equation is in the zero-reference frame. The rotor inertia $(M)$ is the product of the moment of inertia $\left(J \mathrm{in} \mathrm{kg} \cdot \mathrm{m}^{2}\right)$ and the angular velocity $(\omega$ in $\mathrm{rad} / \mathrm{s})$, i.e., $M=J \cdot \omega$. The damping coefficient $D$ represents the damping sources. The terms $P^{(m)}$ and $P^{(e)}$ denote the generator's mechanical input power and the electrical output power, respectively. The terms $\Delta P^{(m)}$ and $\Delta P^{(e)}$ represent the deviation in the respective powers. The electrical dynamics is faster than the mechanical dynamics of the synchronous generator. Therefore, on the transient stability time scale, the deviation in mechanical power is negligible. Making the assumptions: $P^{(m)} \approx P^{(e)}$ and $\Delta P^{(m)} \approx 0$, then (12) is re-written as follows:

$$
M \frac{d}{d t} \omega+D \cdot \omega=-\Delta P^{(e)} .
$$

For a lossless and homogeneous network, consider a node degree equal to $N$, then from Kirchhoff's current law, the electrical power flowing out of the node should equal the sum flowing to the adjacent connected nodes:

$$
P^{(e)}=\sum_{i=1}^{N} P_{i}^{(e)} .
$$

For homogeneous connections $P_{i}^{(e)}=P_{n} \quad ; \forall i$. Then, 


$$
P^{(e)}=N \cdot P_{n}
$$

For a disturbance input which causes a deviation in $P^{(e)}$, the following is assumed for a homogeneous network:

$$
\Delta P^{(e)}=N \cdot \Delta P_{n} .
$$

Then, during a power imbalance disturbance at a node, the frequency dynamics at an adjacent node with degree $N$ is as follows:

$$
M \frac{d}{d t} \omega+D \cdot \omega=-\frac{\Delta P^{(e)}}{N}=-\Delta P_{n} .
$$

Considering a lossless, homogeneous connection and uniform network, then (17) is based on Kirchhoff's current law to describe the disturbance impact dispersion. The impact of a generation/load imbalance disturbance, with a magnitude of $\Delta P^{(e)}$, will be shared with the $N$ directly connected adjacent nodes if an equal power transfer to all these adjacent nodes is assumed (only for homogeneous connections). Therefore, the more neighbours a node has (larger node degree capacity for nonhomogeneous networks), the more support there is to endure the disturbance, which decreases a node's sensitivity to disturbances.

Deferring from the homogeneous susceptance assumption for G1, G2, and G3, the eigenvector components belonging to the algebraic connectivity, are presented in (18)-(29). The analytic derivation results show that the relative stability of a node expands beyond just node degree. The node stability in terms of network topology has a hierarchical form. Node stability also depends on the stability of the connected neighbours. The eigenvector components map to the nodes, then the smaller the eigenvector component magnitude is (compared to the other nodes), the more stable the node of interest. The $u_{0}$ component maps to node 0 . The vector component magnitude of $u_{0}$ is dependent on the $u_{1}$ (node 1 ) vector component magnitude and the susceptance between nodes 0 and 1 .

G1:

$$
\begin{aligned}
& u_{0}=\frac{b_{01} u_{1}}{b_{01}-\lambda_{2}} \\
& u_{1}=\frac{b_{01} u_{0}+b_{12} u_{2}}{b_{01}+b_{02}-\lambda_{2}} \\
& u_{2}=\frac{b_{23} u_{3}+b_{12} u_{1}}{b_{23}+b_{12}-\lambda_{2}} \\
& u_{3}=\frac{b_{23} u_{2}}{b_{23}-\lambda_{2}} .
\end{aligned}
$$

G2:

$$
\begin{aligned}
& u_{0}=\frac{b_{01} u_{1}}{b_{01}-\lambda_{2}} \\
& u_{1}=\frac{b_{01} u_{0}+b_{12} u_{2}+b_{13} u_{4}}{b_{01}+b_{12}+b_{13}-\lambda_{2}} \\
& u_{2}=\frac{b_{12} u_{1}}{b_{12}-\lambda_{2}} \\
& u_{3}=\frac{b_{12} u_{1}}{b_{13}-\lambda_{2}} .
\end{aligned}
$$


G3:

$$
\begin{aligned}
& u_{0}=\frac{b_{01} u_{1}}{b_{01}-\lambda_{2}} \\
& u_{1}=\frac{b_{01} u_{0}+b_{12} u_{2}+b_{13} u_{3}}{b_{01}+b_{12}+b_{13}-\lambda_{2}} \\
& u_{2}=\frac{b_{12} u_{1}+b_{23} u_{3}}{b_{12}+b_{23}-\lambda_{2}} \\
& u_{3}=\frac{b_{13} u_{1}+b_{23} u_{2}}{b_{31}+b_{23}-\lambda_{2}} .
\end{aligned}
$$

Since the eigenvalues of the Laplacian are non-negative, the following is valid for $u_{0}$ :

$$
\frac{b_{i j}}{b_{i j}-\lambda_{2}}>1 \text {. }
$$

Thus, the magnitude of $u_{0}$ is higher than $u_{1}$, which indicates that node 1 is more robust than node 0 in terms of network topology. This result also applies for $u_{3}$ in G1 and G2 (node 3) and $u_{2}$ in G2 (node 2).

Consider $u_{1}$ and $u_{2}$ in G1, which map to nodes 1 and 2, respectively. These nodes "inherit" robustness from their two neighbouring nodes (higher node degree than nodes 0 and 3 in G1). The following is true for $u_{1}$ and $u_{2}$ :

$$
\begin{gathered}
\frac{b_{i j}}{b_{i j}+b_{j k}-\lambda_{2}}<1 \\
\frac{b_{j k}}{b_{i j}+b_{j k}-\lambda_{2}}<1 .
\end{gathered}
$$

Thus, the factor of the contributing vector component is smaller than one. Since the $\lambda_{2}$-eigenvector is orthogonal to the constant vector $\left(\lambda_{1}=0 \rightarrow \mathbb{1} \in \mathbb{R}^{N \times 1}\right)$, the dot product requires that the sum of the elements for the non-constant eigenvectors equal zero. Hence the positive and negative components in the eigenvectors. Therefore, in terms of topology, nodes 1 and 2 are more robust than the boundary nodes in G1. In G2 and G3, the increase in node degree for node 1 further enhances the relative stability of node 1 . So far, the results point to node degree and the node's hierarchical position contributing to its robustness.

\subsection{Impact of Adding a One-Degree Node.}

The next step is to analyse a node's robustness when introduced to a new node. The node assessment uses the spectral method introduced by [25]. The intention is to evaluate the magnitude of the eigenvector components of the smallest non-zero eigenvalue of the network Laplacian. Every network node maps to the $\lambda_{2}$-eigenvector. The size of the vector component indicates the relative robustness of the corresponding node. Small eigenvector elements map to nodes that are less sensitive to network disturbances. These nodes are thus relatively more robust in the network.

The procedure involves evaluating the eigenvector components when adding a node with a single degree to a network with two distinct clusters of different node degree sizes. The network used for the analysis contains 10 nodes (see Figure 2a for network topology). The network graph consists of two clusters, a small cluster centred around node 0 and a larger one centred around node 4 . A new node (with a node degree of one) connects to nodes $0,3,4$, and 9, respectively. An analysis of the eigenvector components for the four cases led to the following observations: 
- $\quad$ Considering the base case (see Figure 2b), before a node addition to the 10-node network, it is noted that the least sensitive nodes contain the higher node degrees (node 0 and 4 ) in each cluster. These nodes have node degrees equal to four and six, respectively;

- $\quad$ The integration of the new node at node 3 (an already present one-degree node in the network) increased the intermediate node's (node 3) sensitivity to network disturbances, see Figure 2c. Although the intermediate node has an increased connection, it has also increased sensitivity. Note that for node 3, its gate-way node to the rest of the network (node 0 ) and the immediate neighbours of (node 0 ) has reduced sensitivity;

- The integration of the new node at node 9 (an already present one-degree node) increased the intermediate node's (node 9) sensitivity to network disturbances, see Figure $2 \mathrm{~d}$. Although the intermediate node has an increased connection, it also increased sensitivity. Note that for node 9, its gate-way node (node 4) to the rest of the network and the immediate neighbours of the gate-way node has reduced sensitivity. Since node 9's gate-way node (node 4 ) has a higher degree than node 0 , and since node 9 facilitates the connection of the new node (with node degree of one), the increased sensitivity for node 9 is still lower than nodes 1,2 , and 3 due to the robustness of node 4;

- $\quad$ Adding a node via a hub node (nodes 0 or 4 ) reduces the sensitivity of all the neighbours of that hub node, see Figure 2e,f. For the case where node 10 connects to node 0 , the amplitude of the eigenvector components associated with the neighbouring nodes decreases from 0.416 to 0.348 (see Figure 2e). The observation is like the case where node 10 connects to node 4 . The eigenvector element amplitudes reduce from 0.27 to 0.236 , for the neighbours of node 4 (see Figure $2 \mathrm{f}$ ). These observations agree with the expression in (17).

From this spectral analysis, the results show that a node's degree and connection to other high-degree nodes determines its relative robustness to disturbances in a network. The robustness of the nodes impacts a network's overall frequency response. It is now simple to identify the stable, and less stable areas in homogeneous-like networks, through the node degree metric. However, for large and complex networks, a node's hierarchical position also has a significant contribution to its robustness. Along with resistance distance (effective impedance between any two nodes/buses), a node's degree and its link to other high-degree nodes influence its sensitivity to network disturbances. This sensitivity to network disturbances indication is on the $\lambda_{2}$-eigenvector of the graph Laplacian.

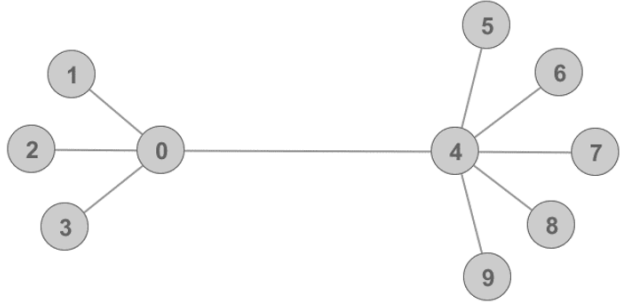

(a) A 10-node network with two clusters.

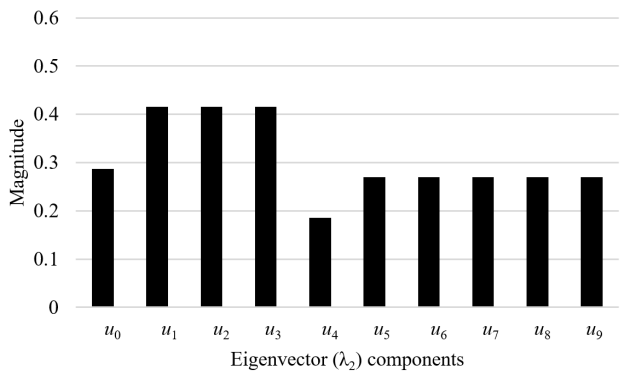

(b) Eigenvector component magnitudes before adding a one-degree node.

Figure 2. Cont. 


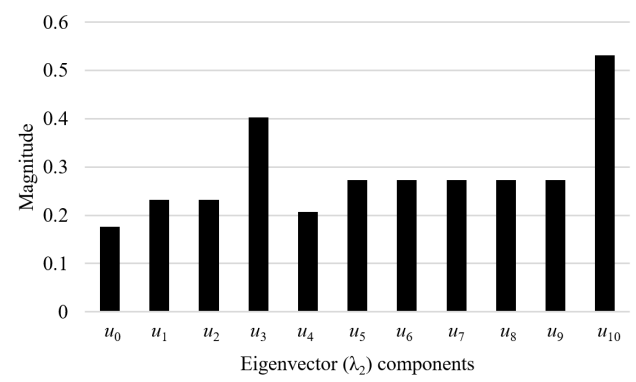

(c) Eigenvector component magnitudes when adding a one-degree node to node 3 .

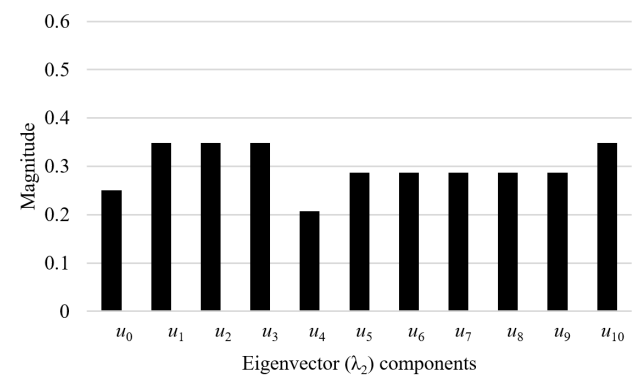

(e) Eigenvector component magnitudes when adding a one-degree node to node 0 .

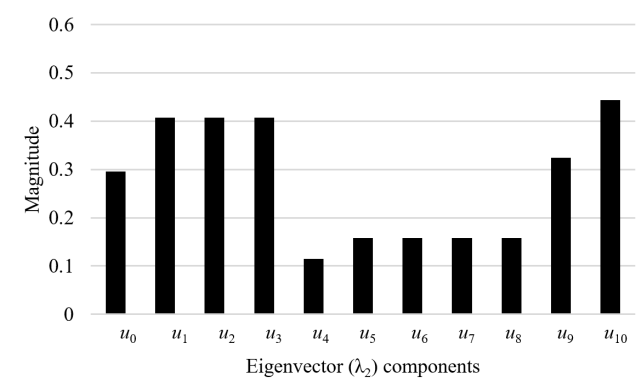

(d) Eigenvector component magnitudes when adding a one-degree node to node 9.

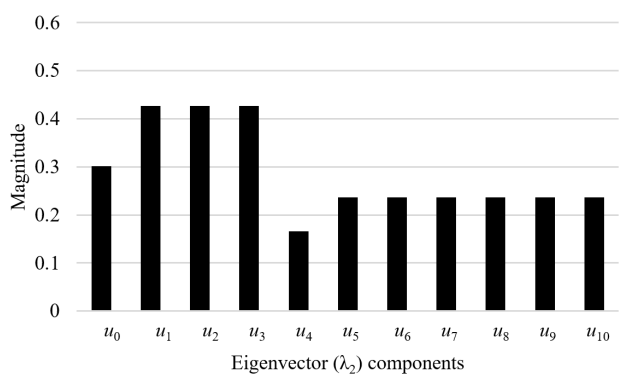

(f) Eigenvector component magnitudes when adding a one-degree node to node 4 .

Figure 2. A 10-node network and the spectral analysis plots. Nodes 0 to 9 maps to $u_{0}$ to $u_{9}$, with $u_{10}$ mapping to the newly-added node.

\section{Disturbance Impact Propagation}

To obtain further insight into how node degree influences the stability of different areas in a network led to investigating electromechanical disturbance propagation in a power system network to find the influence of node degree on the impact flow of a power imbalance disturbance.

\subsection{Simulation Setup}

For the disturbance propagation analysis in this section, we implement three 20node network topologies, where each node represents a distributed generation unit. The topologies selection is such that the influence of node degree is observable where the network splits into multiple branches. Figure 3 illustrates the three network topology graphs. Graph G1 is a bus-topology network, which means all the nodes have a node degree equal to two, except for the boundary nodes, which have a node degree of one. Graph G2 splits into three branches at node 5. Therefore, node 5 has a node degree equal to three. Lastly, graph G3 splits into four branches at node 5, which means node 5 has a node degree of four. In the Digsilent PowerFactory simulation software, the transmission line voltages are $110 \mathrm{kV}$ with $6 \Omega$ reactance between buses. Since the reactance between all adjacent nodes is the same, the term node distance refers to the number of nodes between the disturbed node (node 0 ) and the node of interest. The disturbance location in all three networks is at node 0 . An analysis of the RoCoF in the time and space domains follows the triggering of a low-frequency disturbance event (a relatively small load step input to create a generation/load imbalance disturbance to trigger the small-signal dynamics). 


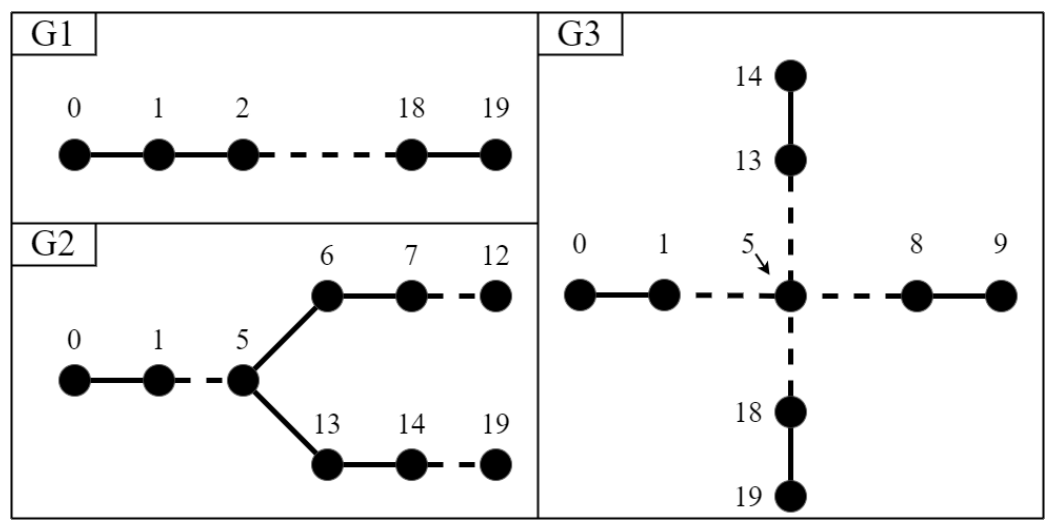

Figure 3. Three network/graph topologies with the network consisting of 20 nodes.

\subsection{Disturbance Behaviour Analysis}

Analysing the low-frequency event for G1, the observation is that the disturbance (RoCoF) propagates as a wave, see simulation output in Figure 4a. As the disturbed generator swings, the generator injects oscillatory disturbances into the network, as shown in Figure 4a. The disturbance propagates to the boundaries of the network. At a boundary node, the wave phase shifts $180^{\circ}$ and propagate backwards, i.e., wave reflection, as seen in Figure $4 \mathrm{~b}$. The counter-propagating waves undergo interference which results in partial standing waves, see Figure 4c.

There is a zero net energy transfer in a pure standing wave, but since our network is not a lossless medium, the result is a partial standing wave, i.e., a combination of a standing wave and a travelling wave. The travelling (transverse) wave component supplies the network losses until all the disturbance energy dissipates to zero. The longer a network branch is, the longer the disturbance wave propagates before it reflects from a boundary node. The longer a network branch is, the further the disturbance wave propagates before it reflects from a boundary. The longer it takes for a standing wave to form, the more time is available for the disturbance energy to dissipate. Thus, the faster the RoCoF magnitude decays in time.

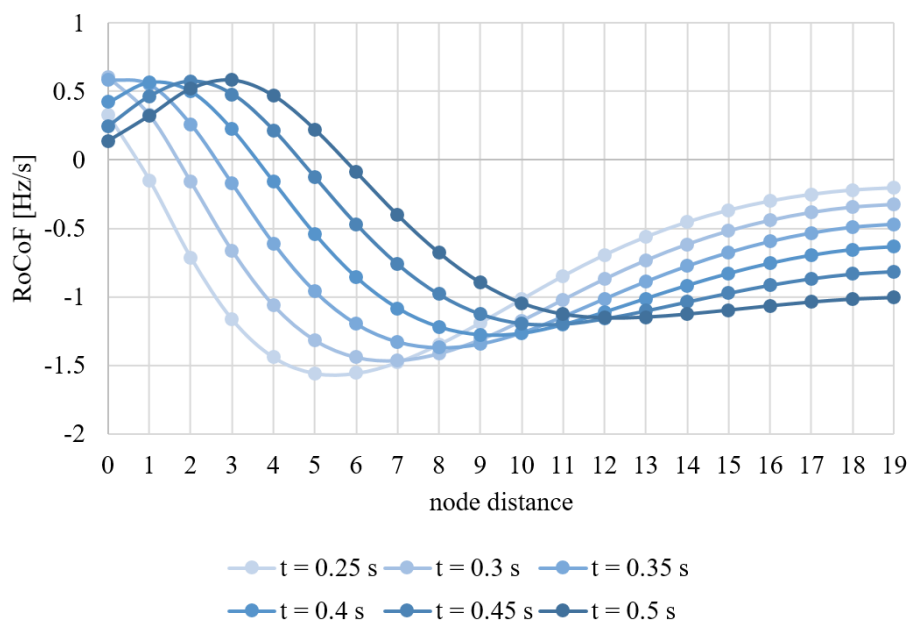

(a) $\operatorname{RoCoF}(\mathrm{Hz} / \mathrm{s})$ propagation (transverse wave).

Figure 4. Cont. 


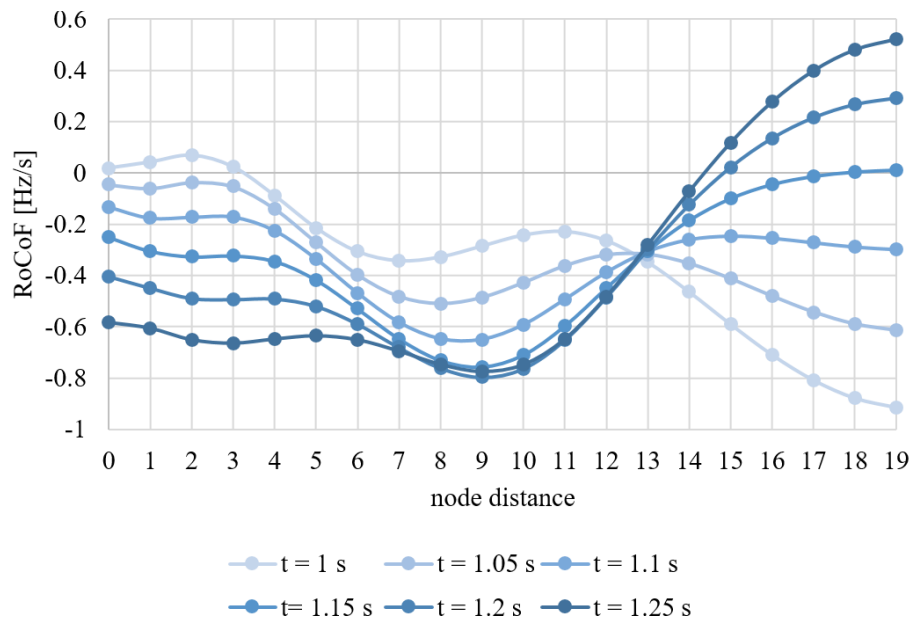

(b) $\operatorname{RoCoF}(\mathrm{Hz} / \mathrm{s})$ wave reflecting (wave reflection).

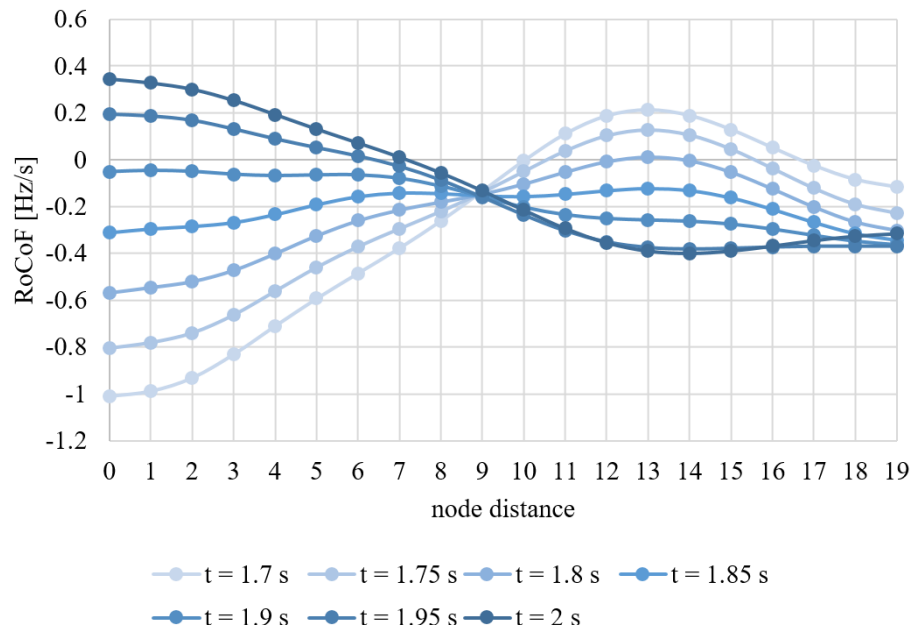

(c) $\operatorname{RoCoF}(\mathrm{Hz} / \mathrm{s})$ standing wave (partial).

Figure 4. Disturbance propagation in a wave-like manner, in network G1, following a disturbance at node 0 (boundary node). (a) Transverse wave, (b) wave reflection, and (c) standing (partial) wave.

The time trajectory of the maximum RoCoF magnitude in G1, G2, and G3, are plotted in Figure 5. Observing the trend for G1, the disturbance amplitude decays as it propagates to node 19. At 0.525 seconds after the disturbance, the wave reaches the boundary and reflects. For both G2 and G3, node 5 splits the network into multiple branches. When the disturbance propagation arrives at node 5 (after 0.2 seconds), a share of the wave energy reflects, and the remainder propagates in the other branches. The disturbance energy propagates forwards and backwards until all the disturbance energy dissipates in the network.

Since the initial disturbance propagation stage for G1 occurs longer than for G2 and G3, the RoCoF magnitude decays on average faster for G1. The disturbance wave in G1 takes longer to reach a boundary node compared to G2 and G3. Thus, more energy gets dissipated before the formation of partial standing waves. Figure 5 shows that G1 has the lowest RoCoF magnitude from 0.525 seconds and onwards. Comparing G2 and G3, graph G2 splits into fewer branches than G3, and the branch lengths in G2 are slightly longer than in G3. Thus, comparing G2 to G3, the slight delay in wave reflection for G2 results in an average faster RoCoF decay. 


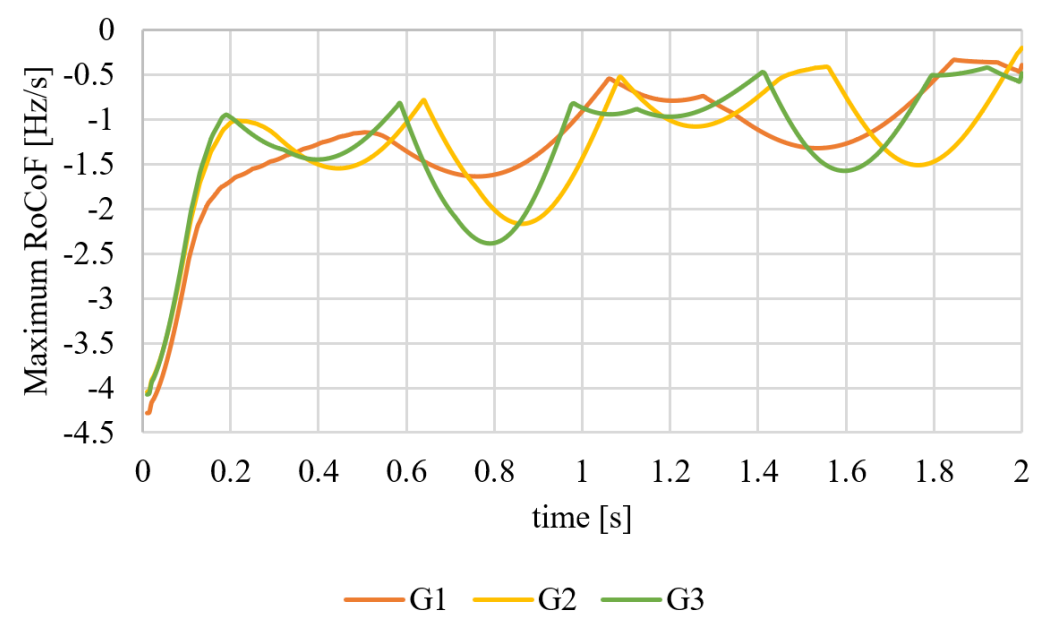

Figure 5. Maximum RoCoF over time for each network in Figure 3.

\subsection{Impact of Node Degree on Disturbance Wave Propagation.}

Understanding the wave-like nature of disturbance impact propagation in a power system network allows for further insight into how a network topology feature, like node degree, influences the propagation of a disturbance in a network. Figure 3 presents the three network graphs simulated to investigate the influence of node degree on disturbance propagation. The only topological difference between the three networks is the node degree of node 5 . The three different network graphs split after the fifth node, from the disturbance location, into a different number of branches. Thus, node 5 in G1, G2, and G3 have node degrees of 2,3, and 4, respectively. Figure 6 shows the RoCoF wave peak position over time for the three networks. The RoCoF peak trajectory is similar for the first four nodes. However, after the network split, i.e., from the fifth node, the wave trajectory significantly differs. It is noticeable from Figure 6 that the RoCoF wave velocities for G2 and G3 are higher post node 5, compared to the RoCoF peak trajectory for G1.

The propagation rate of the RoCoF wave remains consistent for the regions post network split (node 5). However, towards the end of the networks' branches, the wave propagation rate increases again. Based on the simulations, the observation is that the node degree impacts the disturbance propagation. The next step was to analyse the disturbance wave propagation speed for the three topologies.

Figure 7 shows the RoCoF propagation speeds across the spatial dimension of the network. The velocity-over-space plot shows that at node 5, the RoCoF propagation speed temporarily increases. This temporary speed increase is not identical for the three networks. For node 5 in G1, the node degree is the same as the surrounding nodes' degree, and this corresponds with no increase in the RoCoF propagation speed. Node 5 in G2 has a higher node degree than its surrounding nodes. This location is where the RoCoF's propagation speed increases. In G3, node 5 has a higher node degree than the same nodes in G1 and G2, and this corresponds to a significantly higher wave propagation speed in G3.

For mechanical waves, such as a vibrating string, the speed of a transverse wave is:

$$
v=\sqrt{\frac{T_{\mathrm{tsn}}}{\mu_{\mathrm{mass}}}} .
$$

where $T_{\text {tsn }}$ denotes the tension in a string, and $\mu_{\text {mass }}$ represents the linear mass density. Considering any medium for mechanical wave propagation, then in general, the terms $T_{\mathrm{tsn}}$ and $\mu_{\text {mass }}$ represents the restoring and inertial properties of the medium, respectively. Thus,

$$
\text { wave speed }(v)=\sqrt{\frac{\text { restoring property }}{\text { inertial property }}} .
$$




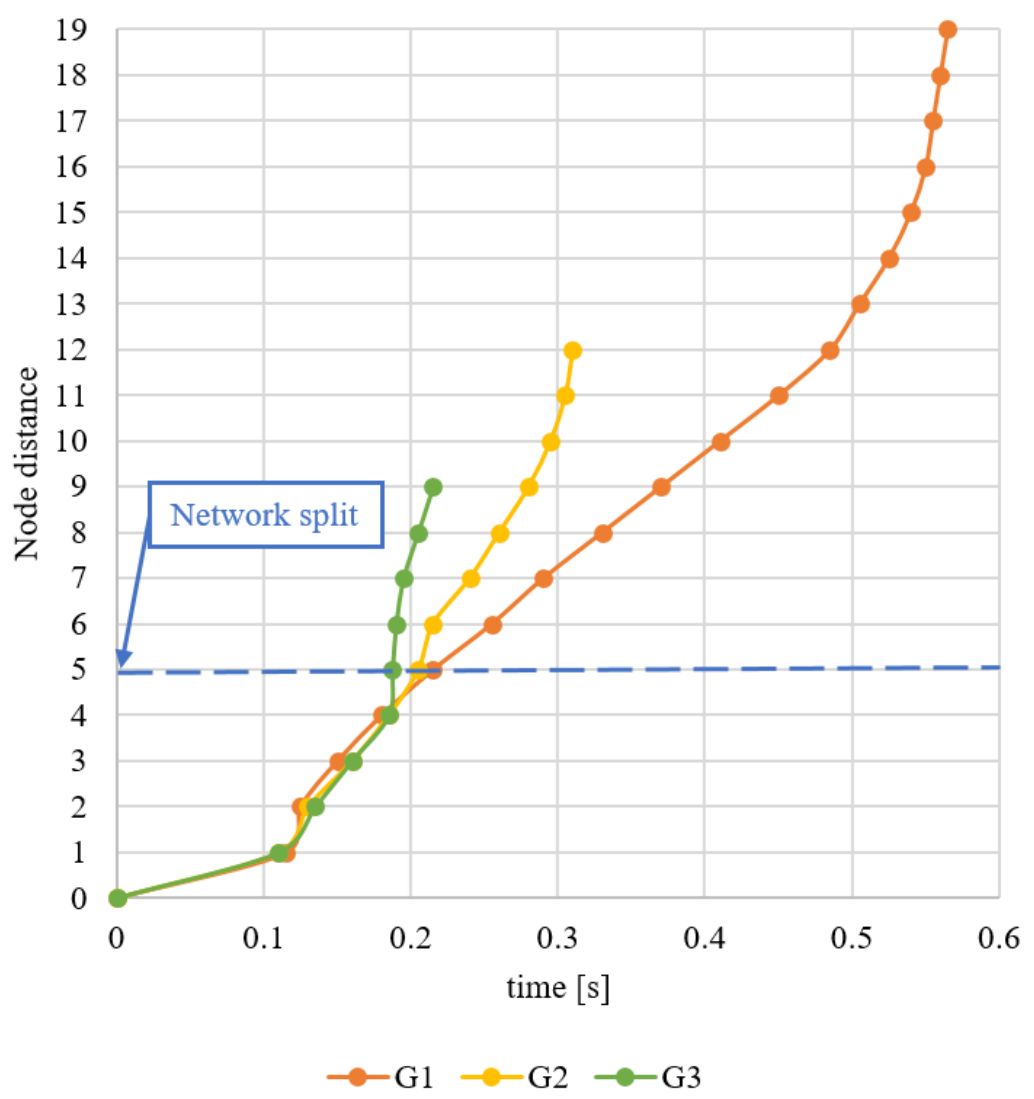

Figure 6. RoCoF wave position with respect to time.

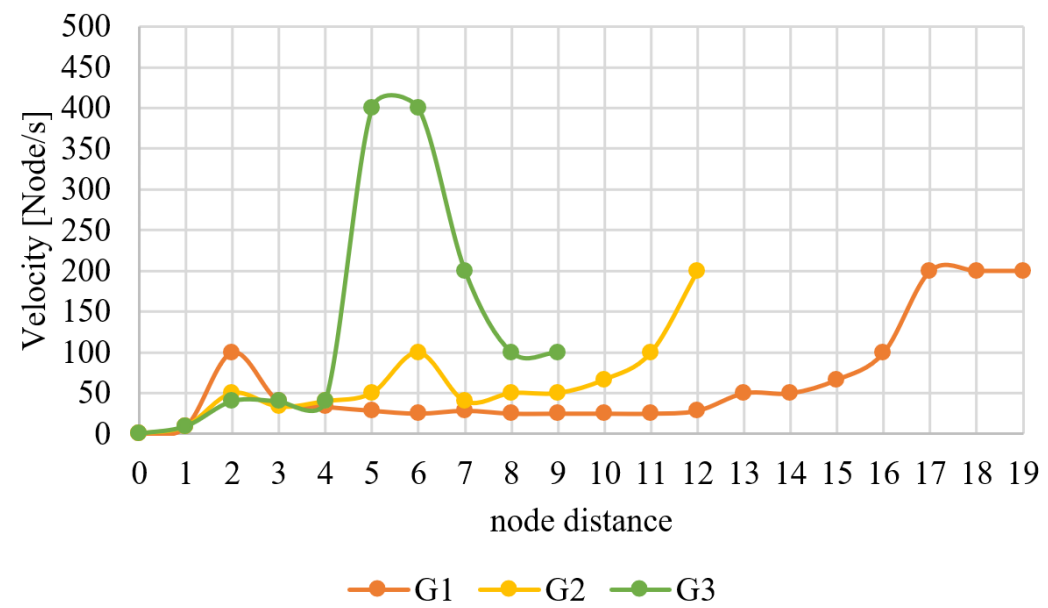

Figure 7. RoCoF wave velocity over space.

The general wave equation is:

$$
\frac{\partial^{2} \xi}{\partial t^{2}}=v \frac{\partial^{2} \xi}{\partial x^{2}}
$$

The variables $t$ and $x$ denote the time and spatial coordinates, respectively. The general solution of $\xi$ is in the form:

$$
\xi(x, t)=F(x-v t)+G(x+v t) .
$$

where $F$ and $G$ are two wave components travelling in opposite directions. For a disturbance wave in a power system network, (37) is analogues to (34), see Appendix A for 
the derivation of (37). The square of the RoCoF propagation speed is proportional to the transmission capacity between nodes $(i, j)$ and inversely proportional to the inertia at the nodes:

$$
v=\sqrt{\frac{\mathbf{K}_{i j}}{\mathbf{M}_{i i}}} .
$$

For nodes with higher degrees (connection capacity), like node 5 in G2 and G3, the ratio between transmission capacity $\mathbf{K}_{i j}$ and inertia $\mathbf{M}_{i i}$ is more significant. Therefore, there is a higher propagation speed $(v)$. Nodes with a higher node degree capacity have a better ability to disperse the disturbance impact. The node has thus a higher "restoring property". The simulation results presented in Figure 7 supports this interpretation of the different propagation speeds. The wave propagation rate increases as it moves towards the periphery of the network branches. As the disturbance wave propagates and passes, the generator rotors swing back and oscillate relative to the synchronous reference frame. At the network boundary, the wave experiences a $180^{\circ}$ phase shift and propagates in the opposite direction. When the RoCoF wave reflects and its propagation aligns with the backward rotor swing, the resistance to propagation (inertia) becomes effectively less. With the transmission capacity unchanged and less inertial effort, the propagation speed increases at the boundary or reflection point. The increase in wave propagation speed is observable in Figure 7 at the peripheral buses.

For mechanical waves, the rate of energy transfer (power) is as follows [28,29]:

$$
P_{\text {wave }}=\frac{1}{2} \mu_{\text {mass }} A^{2} \omega_{\text {wave }}^{2} \frac{\lambda_{\text {wave }}}{T}=\frac{1}{2} \mu_{\text {mass }} A^{2} \omega_{\text {wave }}^{2} v \text {. }
$$

The parameter $\mu_{\text {mass }}$ is called the mass density. In this context, $\mu_{\text {mass }}$ refers to the inertia at each node in a homogeneous inertia network. Let $A$ represent the amplitude of the RoCoF wave. The amplitude and angular frequency $\omega_{\text {wave }}$ of the wave depends on the disturbance input [29]. The wavelength and its period are $\lambda_{\text {wave }}$ and $T$, respectively. The relationship between wave velocity $v$, wavelength, and the wave period is as follows [30]:

$$
\omega_{\text {wave }}=2 \pi \frac{v}{\lambda_{\text {wave }}}
$$

From (38), the power carried by the transverse RoCoF wave is proportional to the propagation speed and the square of the amplitude and angular frequency [31]. Based on the simulation results, the observation is that a higher node degree enables faster propagation speeds due to the higher transmission capacity $\left(\mathbf{K}_{i j}\right)$. A node whose degree is more than two is a branch intersection. When a wave enters the branch split node, the wave splits to propagate along the different paths. The disturbance propagation wavelength is shorter in networks with branch intersections than in a bus-topology network of the same network size. Thus, the impact of a power imbalance disturbance is more suppressed in networks with a higher average node degree. This conclusion explains why the tree topology is optimal for noise reduction [26].

The maximum RoCoF wave amplitudes for G1 to G3 are plotted and compared over the spatial and time domains in Figure 8a,b, respectively. The observation for the spatial dimension is that the maximum RoCoF magnitudes at the boundary node for graphs G1 to G3 are approximately the same. However, there is a significant difference for the nodes located between boundary nodes. The RoCoF wave amplitude reaches a minimum at the central location of the networks. Comparing the networks with branch splits at node 5 (G2 and G3) with G1 (which is a single branch), then the observation is that the RoCoF wave amplitude significantly decreases at node 5 (higher degree node). Thus, based on (38), the impact of the disturbance is comparably less at node 5 (and the surrounding nodes) than at the boundary nodes (and their closest neighbours) and these results support (17) and the findings from Section 2. 


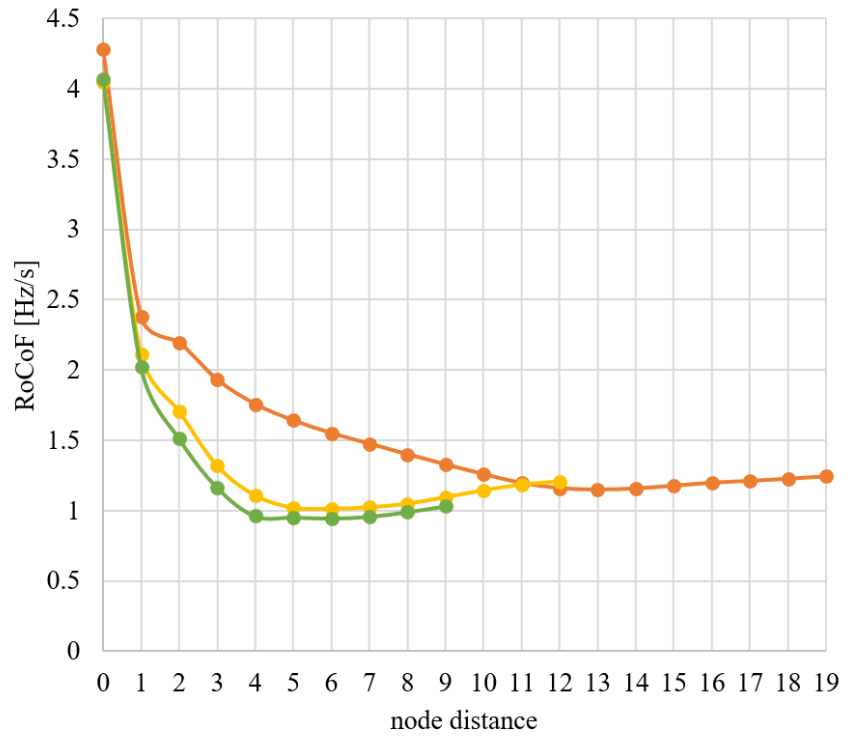

$\multimap-\mathrm{G} 1 \multimap-\mathrm{G} 2 \multimap-\mathrm{G} 3$

(a) Maximum RoCoF wave magnitude over space.

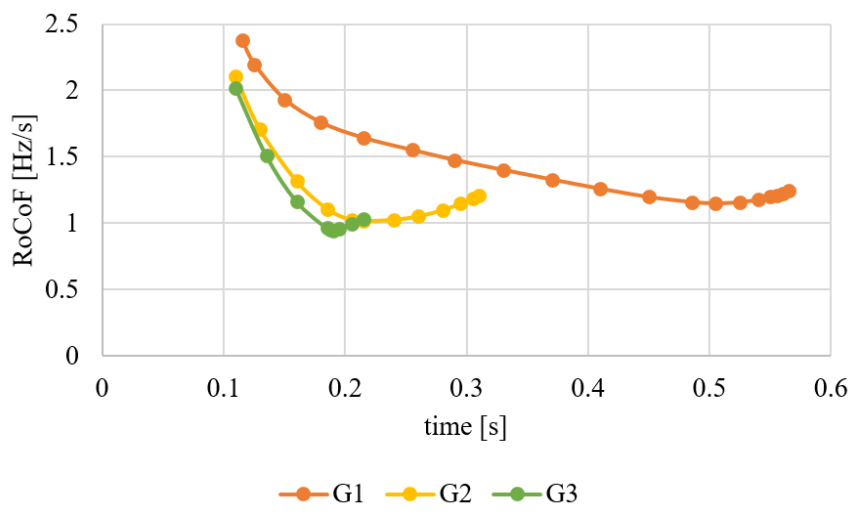

(b) Maximum RoCoF wave magnitude over time.

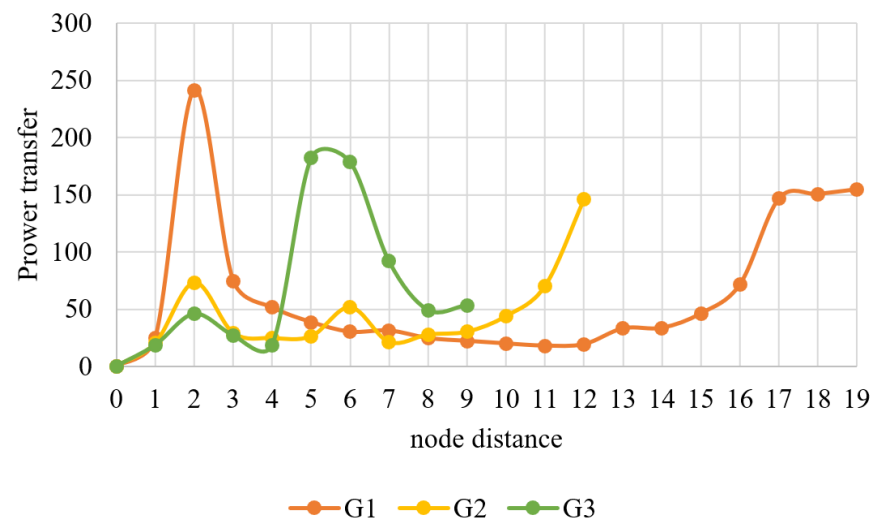

(c) The power transferred by the RoCoF wave proportional to the wave speed $(v)$ and magnitude squared $\left(A^{2}\right)$.

Figure 8. Disturbance wave parameter plots. (a) RoCoF magnitude versus space (position), (b) RoCoF magnitude versus time, and (c) Rate of energy (power) transfer of the wave. 
The wave power is proportional to the square of the amplitude and angular frequency (38). The wave power is also proportional to the wave speed, however, to a lesser extent than amplitude and angular frequency:

$$
\begin{aligned}
& P \propto A^{2} \\
& P \propto \omega_{\text {wave }}^{2} \\
& P \propto v .
\end{aligned}
$$

The scale of the influence that node degree has on wave speed is larger (Figure 7) than the RoCoF amplitude decay (Figure 8a). Figure 8c presents the power transfer profile for graphs G1 to G3 to illustrate the impact of node degree on the rate of disturbance energy dissipation. Note that the plot does not indicate the absolute value of power transfer but only indicates the dominating impact of wave speed on the power transfer due to the node degree.

From Figure 8c, the following observations are made:

- $\quad$ The disturbance starts at node 0 . The disturbance amplitude is the highest at node 0 , as indicated in Figure 8a. However, initially, the impact of the disturbance is only felt locally, and the impact flow is approximately stationary, as shown in Figure 7. Therefore, the power transfer is close to zero;

- The impact of the disturbance starts to propagate from node 0 to node 1 . As the amplitude is still relatively high but rapidly decreasing, the power transfer increases significantly between nodes 1 and 2;

- From node 5, the topology of the graphs starts to differ. The increase in propagation speed is due to the higher node degree. Note that the propagation speed dominates the change in amplitude. The increased propagation speed increases the energy transfer of the RoCoF wave, which results in a lower disturbance impact at node 5, and its neighbouring nodes. Thus, the disturbance impact is shared with the neighbour nodes, as stated by (17) in Section 2;

- As the wave approaches the far end of the network (boundary), the wave experiences a $180^{\circ}$ phase shift before it reflects and propagates in the opposite direction, which results in partial standing waves. Thus, energy transfer becomes significantly inhibited, and the rest of the disturbance energy gets dissipated through electromechanical and network transmission losses.

\section{Testing on IEEE-14 and IEEE-39 Bus Power Systems.}

The investigation of power system network topology through spectral analysis mainly involved homogeneous networks. Although homogeneous networks do not represent realistic power systems, it provides analytical simplicity and insight into complex structures. In this section, the focus changes from a homogeneous network to a practical power system network. In this section, the IEEE-14 bus and IEEE-39 bus networks (see Figures 9 and 10) serve as test networks for our analytical findings.

The main differences between homogeneous networks and a realistic power system network are the transmission line reactance and the inertia distribution. Node degree is a few orders of magnitude smaller than transmission line reactance, which is typically 0.3 to $0.5 \mathrm{Ohm}$ per kilometre. Therefore, instead of using just the simple node degree metric, the graph Laplacian's diagonal elements are used since the diagonal contains the sum of the connection capacities. This "node degree capacity" serves as a weighted node degree.

Two load step disturbances were simulated on the IEEE-14 bus network at buses 14 and 12 , respectively. In realistic transmission networks, the reactance is more significant than the resistance. Therefore, in this context, resistance distance refers to the effective reactance between any pair of buses. Figure 11 presents the resistance distances from each bus to the disturbance bus, the node degree capacities, and the maximum RoCoFs. 


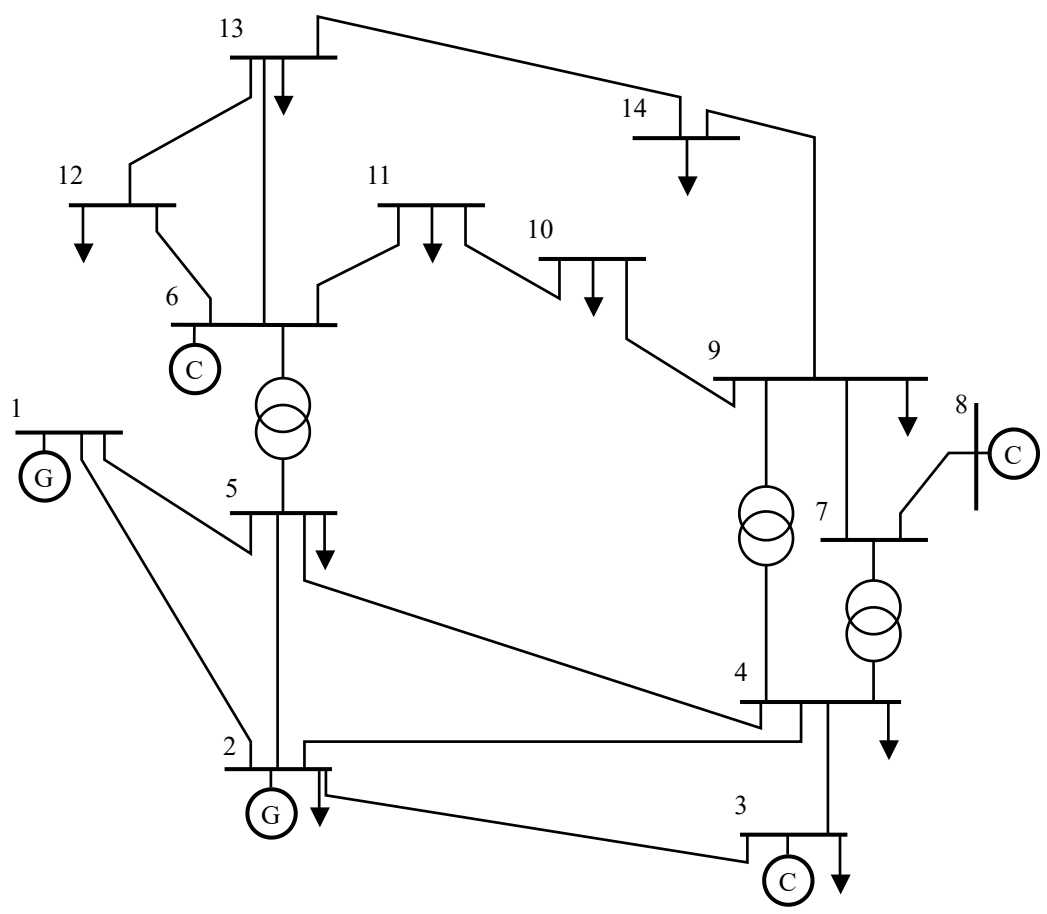

Figure 9. Single-line diagram of the IEEE-14 bus network.

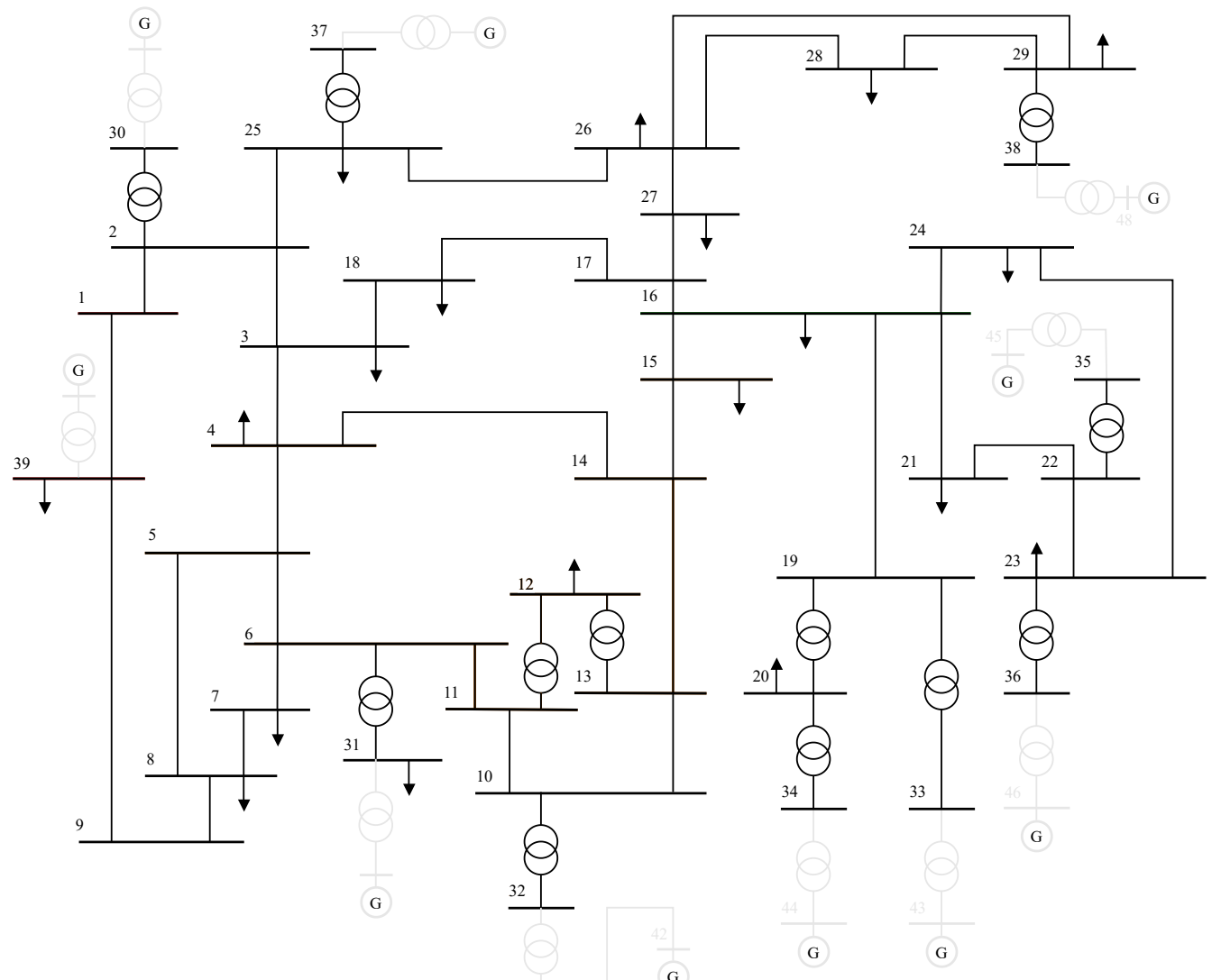

Figure 10. Single-line diagram of the IEEE-39 bus network. 


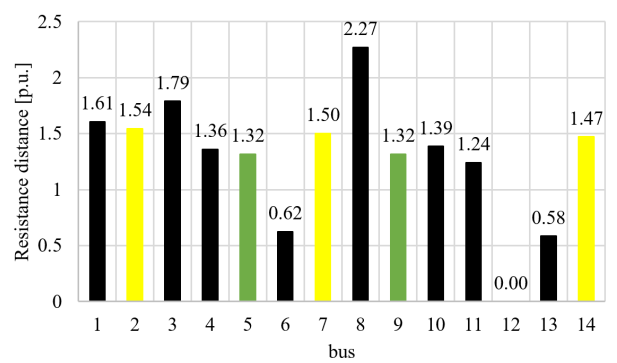

(a) Plot of the resistance distances to bus 12 .

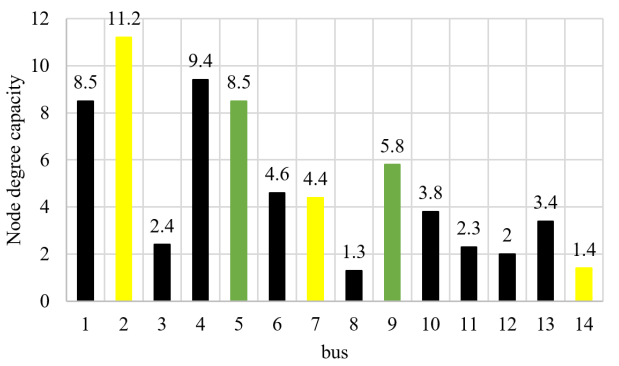

(c) Node degree capacity of each bus.

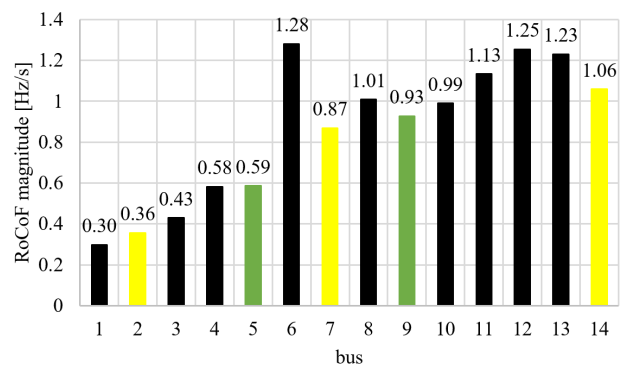

(e) RoCoF magnitudes due to imbalance disturbance at bus 12 .

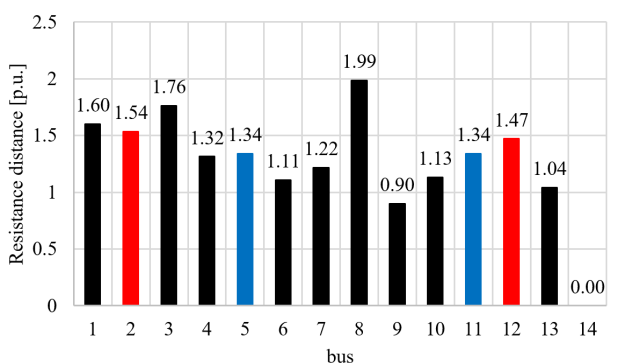

(b) Plot of the resistance distances to bus 14 .

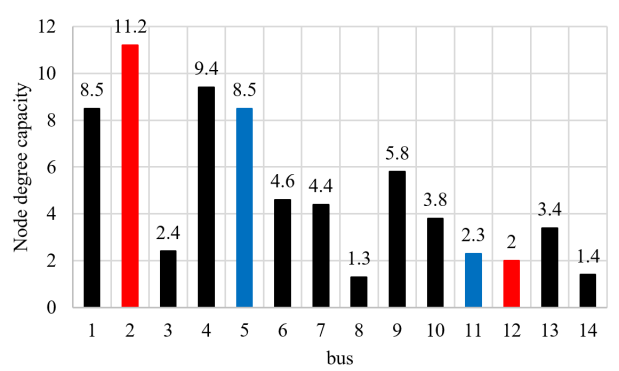

(d) Node degree capacity of each bus.

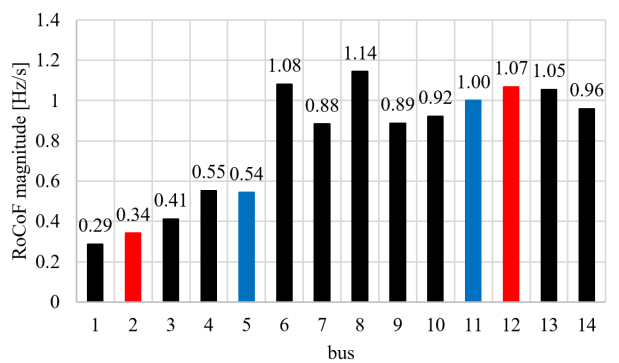

(f) RoCoF magnitudes due to imbalance disturbance at bus 14

Figure 11. IEEE-14 bus: The resistance distances, node degree, and RoCoF plots for disturbances at bus 12 (left-side) and bus 14 (right-side), respectively.

Figure 11a,b show the resistance distances (in per-unit [p.u.]) for each bus to the disturbed bus. Some buses with similar resistance distances are colour coded, e.g., for the disturbance at bus 14 scenario, the bus pair $(2,12)$-red, $(5,11)$-blue have similar resistance distances but are at different locations. The node degree capacity of each bus is plotted in Figure $11 c, d$, respectively. The node pair colour codes link to those in Figure 11a,b. Finally, the maximum RoCoF magnitude recorded at each bus is in Figure 11e,f for the respective disturbance locations.

Following the colour-coded pairs from the resistance distances, the node degree capacities, and comparing the node degree to the RoCoF plots reveals a trend. Comparing the buses with identical resistance distances shows that the buses with the greater node degree capacities experience a smaller RoCoF.

In the case of the IEEE-39 bus simulations, the same observations are made from the simulation results and analysis as shown in Figure 12. 


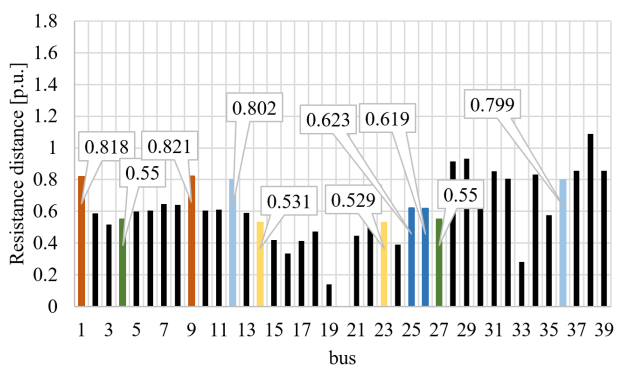

(a) Plot of the resistance distances to bus 20 .

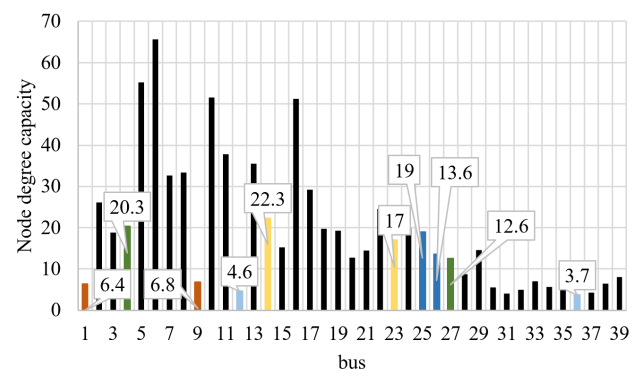

(c) Node degree capacity of each bus.

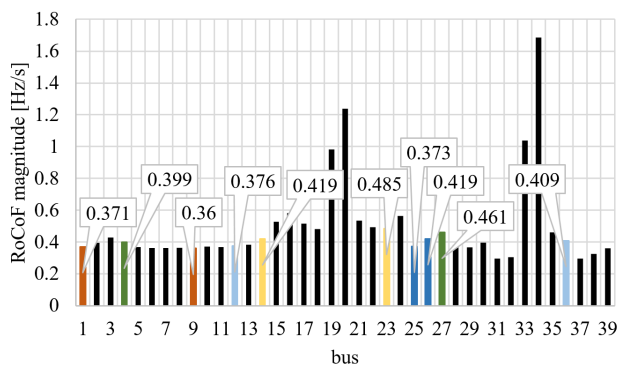

(e) RoCoF magnitudes due to imbalance disturbance at bus 20 .

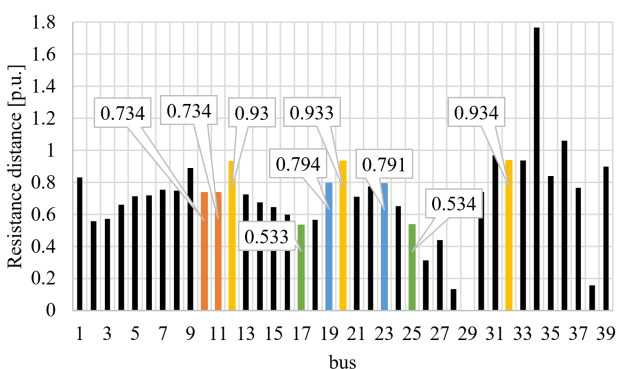

(b) Plot of the resistance distances to bus 29.

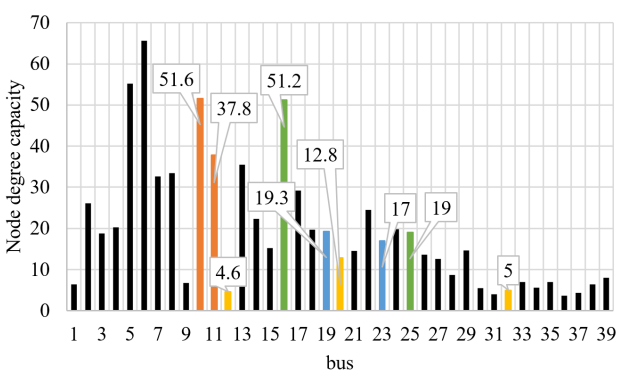

(d) Node degree capacity of each bus.

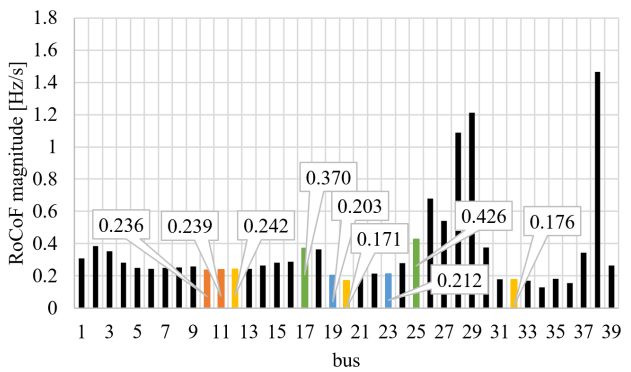

(f) RoCoF magnitudes due to imbalance disturbance at bus 29 .

Figure 12. IEEE-39 bus: The resistance distances, node degree, and RoCoF plots for disturbances at bus 20 (left) and bus 29 (right), respectively.

The IEEE-14 bus is smaller than the IEEE-39 bus network, and for the larger of the two networks (IEEE-39 bus network), the impact of the resistance distance becomes more significant than the node degree capacity measure, and thus, the difference in RoCoFs are less between buses with similar resistance distances from the disturbance location. The simulation results are, however, consistent and in agreement with the theoretical findings. Therefore, the practical network simulations confirm the analytical findings, based on the graph spectral analysis, on node stability.

\section{Discussion}

A generation/load imbalance disturbance triggers the transient dynamics of the synchronous generators' rotors and network buses' voltage phase angle. The result is a deviation from the steady-state power flow, which leads to an excursion in the power system frequency. For a given power system network, an imbalance disturbance of a sufficient size can lead to the system dynamics leaving the basin of attraction of the synchronous state [32]. In [32], the authors define a 1-solitary state as the loss of synchronism of a single generator in a power system network. The formation of a solitary state tends to create a chain reaction by desynchronising the neighbouring buses [32]. Frequency transients of sufficient magnitude result in the activation of distance protection leading to 
network splitting, further promoting the chain reaction of instability, ultimately leading to a system-wide blackout [14]. Since the influence of changing network topology on distance protection settings was not the focus of this study, the reader is encouraged to read the more detailed work of M. Tasdighi and M. Kezunovic $[15,16]$ on distance protection for changes in network topology due to a disturbance.

Recent literature on low-inertia power systems indicates that frequency stability depends on the power system network topology, node dynamics, and coupling strength $[9,18,21]$.

Analysing the graph's spectrum of small and homogeneous network structures reveals the following: A node's degree, and its link to other high degree nodes, influences its robustness. The node degree determines the magnitude of the corresponding $\lambda_{2}$-eigenvector component. Nodes with a higher degree have a small $\lambda_{2}$-eigenvector component associated with them and thus is relatively more robust. Since the node dynamics (bus frequency) couples through the power flow, we show that the node robustness depends on the stability of the power flow through the node/bus. The power flowing through a bus is more diverse for a higher number of branches (edges) entering or leaving that bus. Therefore, the power flow is more robust, and the frequency response follows a more stable trajectory. The stability of higher degree nodes is from the steady power flow due to numerous and diverse paths (edge connections) and the support (inertial response) from neighbouring nodes. Connectivity also enhances the dispersion of the disturbance energy.

It is now simple with the node degree measure to identify the stable and less stable areas in homogeneous networks. However, for larger and more complex network systems, a node's hierarchical position-i.e., its connection to other high degree nodes-also determines its sensitivity. The eigenvector belonging to the smallest non-zero eigenvalue, which maps to the network nodes, indicates the relative robustness through the vector element's magnitude [25].

When considering network expansion for a low-inertia power system, the investigation in Section 2.1 shows that the integration of a one-degree node destabilises the node facilitating the integration if the node is not relatively robust. However, when the new node integrates through a high degree hub (node with a relatively high degree), the stability increases. An alternative is to integrate through a node with a connection to a high degree node. Thus, receiving stability through a hierarchical position.

To obtain further insight into how node degree influences the stability of different areas in a network led to investigating electromechanical disturbance propagation in a power system network. The simulation of three network topologies with a different number of branches (a different node degree at intersection node), but the same network size (20 nodes), allowed us to observe the power imbalance impact flow. The observation is that the disturbance propagates through the network in a wave-like manner, carrying the disturbance energy across the entire network. Similar findings of disturbance propagation are found by J. S. Thorp et al. [24,27] using a continuum model. The disturbance propagation has the same characteristics as mechanical waves. The disturbance propagates until it reaches a boundary node, reflects, and creates partial standing waves due to the continuous oscillation feed-in from the perturbed node. Standing waves do not transmit energy. Therefore, the realisation of partial standing waves slows down the decay of the RoCoF magnitude. Another similarity with mechanical waves is that the disturbance wave speed is proportional to the electromechanical medium's restoring property (transmission capacity-the force internally transmitting the wave [29]) and inversely proportional to inertial property (rotational inertia). The derivations in $[24,27,33]$ agree with our result on the propagation velocity (37)—see Appendix A for the derivation. The derivation and simulation results indicate that the RoCoF propagation speed increases at branch intersections (nodes with higher degree capacity) due to the higher transmission capacity (restoring property). The robustness of a high degree node is due to the diverse power flow paths and being better able to disperse the disturbance energy. The results led to further insight into the role of network topology in frequency transient stability. 
Wave Theory provides a framework for understanding the behaviour of disturbance propagation and the influence of the network topology on a global level, like the continuum models in $[24,27,34]$.

The velocity (37) of the disturbance propagation shows that the propagation rate is faster in low-inertia systems when compared to high-inertia power systems. This conclusion agrees with [27]. The disturbance propagation rate is also higher for centralised synchronous generation networks compared to distributed synchronous generation networks. In terms of low-inertia power systems, mitigations include increasing the local inertia using real or virtual inertia and an optimal inertia placement strategy. Therefore, the optimal use of inertial support units in a low-inertia power system is to distribute the inertia throughout the network to slow down the disturbance propagation. The distribution of inertia units in low-inertia power systems can be advantageous to frequency transient stability as shown in [35].

In attempts to find the critical nodes in a network, the authors of [7] conclude that the resistive closeness centrality (closeness centrality) is relevant. In [36], the conclusion is that the further the distance from the disturbance location is, the later in time and weaker the impact is. The authors of [17] found that the peripheral nodes (typical nodes with low resistive centrality) generate the furthest transient excursion when perturbed, which agrees with this research work.

Based on the literature, the combination of resistance distance and centrality is pertinent to identify critical nodes. Since resistance distance is the effective impedance between a pair of nodes in a network $[17,37,38]$, the resistance distance decreases with the number of paths between nodes. Hence, there is a connection between resistance distance and node degree. There is also a link between node degree and resistance centrality due to the higher connectivity. Therefore, it is reasonable to state that the resistive centrality benefits from a higher node degree. However, in practical systems like the IEEE-14 bus network, the order of magnitude for resistance distance is higher compared to the node degree. Thus, the lower order of magnitude for node degree under-represents its contribution to node stability in the family of topological indices. Therefore, in nonhomogeneous power system networks, the node degree capacity is rightly applicable.

For nonhomogeneous transmission line impedances, the spectral analysis results, (18)-(29), indicate that the influence of a neighbouring node-on-node robustness increases for higher susceptance between the node pair (the fraction coefficient approaching one). The opposite is true for transmission line susceptance tending towards zero, with the fraction coefficient approaching zero.

The IEEE-14 and IEEE-39 bus networks served as test cases for the impact of node degree on frequency stability. Although the resistance distance significantly contributes to the frequency stability (also seen from analytical results), it does not carry all the explanatory power. Analysing the RoCoF from nodes with similar resistance distances to the disturbance, the observation is that the nodes with a higher degree capacity indicate a smaller RoCoF. Hence, they are more stable nodes. The bus with the larger node degree capacity shows to be the more frequency-stable bus.

Research on whether noise filtering can be hard-wired in the network architecture found that the optimal network topology is the tree topology [20]. For spatially uncorrelated noise, the noise input is specific and unique to a local area or node. The results of [26] suggest that the optimal noise-cancelling network strives for dense connections with similar strength to provide many alternative paths/routes to and from a given node. The dense connections allow the fluctuations to be smoothed away by averaging the flow across many connections and inputs [26]. These suggestions are in line with the findings of this research.

The results of $[20,26]$ agree in terms of the impact of network topology on disturbance propagation. The research work in [19,39] supports the finding of this work on node degree and node stability. U. Markovic et al. [19] saw that the reduction in network connectivity increases the dependence on synchronous generators for transient stability. 
Direct connections also improve the synchronisation between generators. D. Barmpoutis and R. M. Murray [39] also state that the nodes with large edge densities are robust.

The authors of $[25,40,41]$ have illustrated that the location of inertia is important, and if not spatially distributed correctly, the frequency stability could degrade [42]. In [25], the conclusion is that removing system inertia from the areas (nodes) mapped to the higher magnitude components of the eigenvector belonging to the smallest non-zero eigenvalue of the network Laplacian results in significantly higher RoCoFs compared to the inertia removal from the nodes related to the smaller eigenvector components. A disturbance on the network propagate to remote areas in the system [33], and generally, the larger size eigenvector components belong to the peripheral nodes of a network. Since high degree nodes are the more robust nodes, and due to their high connectivity, these research findings suggest that the optimal placement of inertia involves using the peripheral nodes in a network. An alternative to combat low inertia is to increase the connection of the less robust/stable nodes. This increase in connectivity reduces the sensitivity to disturbances and increases the network's frequency stability.

These research results imply that flow is more regular/stable for highly connected nodes following a disturbance event. Therefore, the inertial response is low compared to a node where the change in power flow is more significant (lower node degree or peripheral nodes). A more substantial deviation in power flow will trigger an equivalent inertial response. Therefore, higher utilisation of the inertia occurs at a lower degree node, typically peripheral nodes in a power system network.

The findings here complements and contributes to the literature mentioned in the introduction [17,24-27]. For passive noise-cancelling network topologies (H. Ronellenfitsch et al. [26]), node degree and reactance distance are the key ingredients for sparse and hierarchical structures. The finding of S. Bhela et al. [20] on the tree topology as a good candidate for noise reduction is a prime example of an optimal mix between network connectivity and desynchronisation through the optimal use of node degree and reactance distance. Regarding increasing network complexity [17], as well as robust network design and stability analysis $[6,19]$, the node degree serves as a rough preliminary and quick identification of relative robustness of network locations. Optimal placement/distribution of inertial response units are thus intuitively approximated based on the least robust network locations. This research also provides further intuitive insight behind the findings in [25] by identifying the topology measures responsible for the eigenvector component magnitudes.

\section{Conclusions}

Along with resistance distance, a node's degree and its link to other high degree nodes influence its sensitivity to network disturbances. A network's overall frequency robustness stems from the sub-areas in a network. It is now simple to identify frequency stable and less stable areas in simple homogeneous network structures. However, for large and complex network topologies, the hierarchical position (its connection to other robust nodes) also plays a role in its sensitivity to network disturbances. From the graph/network Laplacian, the eigenvector of the smallest non-zero eigenvalue indicates a node's sensitivity. From a network topology perspective, we show that the node degree and the resistance distance determine local robustness. We link node degree to how network topology impacts the frequency stability in a power system through Wave Theory. The node degree metric influences the propagation velocity of a network disturbance and thus the RoCoF, of the frequency response, in the time and space domains. The disturbance impact propagation velocity and network path length determine how soon standing waves manifest. Thus, the topology contributes to how fast the energy induced by a network disturbance dissipates to zero.

Author Contributions: Conceptualisation, W.J.F.; methodology, W.J.F.; software, W.J.F; validation, W.J.F.; formal analysis, W.J.F.; investigation, W.J.F.; resources, W.J.F. and A.J.R.; data curation, W.J.F.; writing—original draft preparation, W.J.F.; writing—review and editing, W.J.F. and A.J.R.; visualisa- 
tion, W.J.F.; supervision, A.J.R.; project administration, W.J.F. and A.J.R.; funding acquisition, W.J.F. and A.J.R. All authors have read and agreed to the published version of the manuscript.

Funding: This research was funded by the Centre for Renewable and Sustainable Energy Studies (CRSES) at Stellenbosch University.

Institutional Review Board Statement: Not applicable.

Informed Consent Statement: Not applicable.

Data Availability Statement: Not applicable.

Conflicts of Interest: The authors declare no conflict of interest.

\section{Appendix A. Wave Equation for Rocof Propagation.}

In this section, the disturbance/RoCoF propagation speed (37) is derived. Consider a bus-topology network with $N$ nodes. The frequency dynamics at node/bus $i$ is described by the Swing Equation:

$$
M_{i} \frac{d}{d t} \omega_{i}+D_{i} \omega_{i}=\left(P_{i}^{(m)}+\Delta P_{i}^{(m)}\right)-\left(P_{i}^{(e)}+\Delta P_{i}^{(e)}\right) .
$$

In the synchronously rotating reference frame, $\omega_{i}$ denotes the angular frequency deviation at node $i$ in radians per second [rad/s]. The inertia and damping are denoted by $M_{i}$ and $D_{i}$, respectively. The right side depicts the power balance with the superscript $(m)$ denoting the input power, and $(e)$ denotes the electrical output power. The power balance is split into two components, the average power $P_{i}^{(m)}$ and $P_{i}^{(e)}$ and a fluctuating components $\Delta P_{i}^{(m)}$ and $\Delta P_{i}^{(e)}$. For analytical simplicity, the following assumptions are made:

- $E\left\{P_{i}^{(m)}\right\}=E\left\{P_{i}^{(e)}\right\}$;

- The mechanical dynamics of a synchronous generator is slower than the electrical dynamics of the network. Thus, considering the inertial response time window $\Delta P_{i}^{(m)} \approx 0$;

- $E\left\{\Delta P_{i}^{(e)}\right\}=0$, however, the variation of $\Delta P_{i}^{(e)}$ is not zero $\left(\sigma^{2} \neq 0\right)$;

- Lastly, since the maximum RoCoF occurs immediately after the disturbance and the frequency deviation is small, the term $D_{i} \omega_{i} \approx 0$ for all $i$.

Thus, (A1) is re-written as:

$$
M_{i} \frac{d}{d t} \omega_{i}=-\Delta P_{i}^{(e)}
$$

Equation (A2) states that for a positive deviation in electrical power the RoCoF is negative. Thus, the frequency decreases for a positive load step input and the frequency increases for a negative load step input.

Using the power versus phase angle curve as shown in Figure A1 gives an expression for the electrical power deviation (A3). The transmission capacity is $K_{i j}=\frac{\vee_{i} \vee_{j}}{X_{i j}}$, where the transmission line voltage and reactance are $\vee$ and $X_{i j}$, respectively. The electrical power deviation $\Delta P^{(e)}$ expression is as follows:

$$
\Delta P_{i j}^{(e)}=K_{i j} \sin \left(\delta_{i}+\Delta \delta_{i}-\delta_{j}-\Delta \delta_{j}\right)
$$

- $\Delta \delta_{i j}=\delta_{i}-\delta_{j} ;$

- $\kappa_{i j}=\Delta \delta_{i}-\Delta \delta_{j}$ 


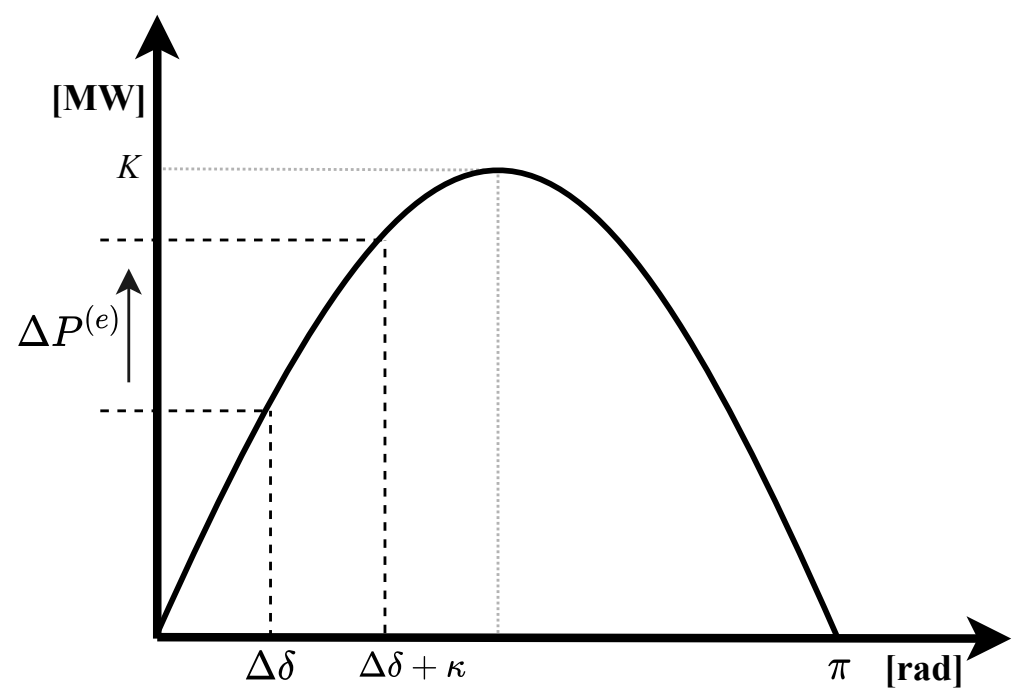

Figure A1. Power angle diagram.

$$
\Delta P_{i j}^{(e)}=K_{i j} \sin \left(\Delta \delta_{i j}+\kappa_{i j}\right)
$$

For large reactance $\left(X_{i j}\right), \Delta \delta_{i} \gg \Delta \delta_{j}$ :

$$
\therefore \kappa_{i j} \approx \Delta \delta_{i}
$$

Then (A4) becomes,

$$
\Delta P_{i j}^{(e)}=K_{i j} \sin \left(\Delta \delta_{i j}+\Delta \delta_{i}\right) .
$$

Substituting (A5) back into (A2), the phase angle dynamics for bus $i$ becomes:

$$
M_{i} \frac{d^{2}}{d t^{2}} \Delta \delta_{i}=-K_{i j} \sin \left(\Delta \delta_{i j}+\Delta \delta_{i}\right)
$$

The following approximation is helpful to find $\delta_{i}$, assuming small-angle deviation:

$$
\sin \left(\Delta \delta_{i j}+\Delta \delta_{i}\right) \approx\left(\Delta \delta_{i j}+\Delta \delta_{i}\right)
$$

The approximation leads to the following second-order nonhomogeneous differential equation:

$$
M_{i} \frac{d^{2}}{d t^{2}} \Delta \delta_{i}+K_{i j} \Delta \delta_{i}=-\mathbf{K}_{i j} \Delta \delta_{i j}
$$

The roots of of the homogeneous part of the characteristic equation are complex $\left(\lambda_{1,2}^{\text {root }}=\mathbf{R e} \pm j \mathbf{I m}\right)$; the real part is equal to zero $(\mathbf{R e}=0)$, and the imaginary part is $\operatorname{Im}=\sqrt{K_{i j} / M_{i}}$.

The solution for this case equates to the negative of the initial disturbance input, i.e., $y_{p}=-\Delta \delta_{i j}$. The general solution for the phase angle dynamics is thus:

$$
\Delta \delta_{i}(n, t)=C_{1} \cos \left(n+\frac{K_{i j}}{M_{i}} t\right)+C_{2} \sin \left(n+\frac{K_{i j}}{M_{i}} t\right)-\Delta \delta_{i j} .
$$

The RoCoF is the second time derivative of (A8). Thus,

$$
\frac{\partial^{2}}{\partial t^{2}} \Delta \delta_{i}(n, t)=\dot{\omega}_{n}
$$




$$
\dot{\omega}_{n}=\left[\frac{K_{i j}}{M_{i}}\right]^{2}\left[-C_{1} \cos \left(n+\frac{K_{i j}}{M_{i}} t\right)-C_{2} \sin \left(n+\frac{K_{i j}}{M_{i}} t\right)\right] .
$$

The general wave equation is:

$$
\frac{\partial^{2} \xi}{\partial t^{2}}=v \frac{\partial^{2} \xi}{\partial x^{2}}
$$

while the general solution is of the form:

$$
\xi(x, t)=F(x-v t)+G(x+v t) .
$$

Equation (A9) is partially in the form of (A10). Thus, the next step is to find if:

$$
\left[-C_{1} \cos \left(n+\frac{K_{i j}}{M_{i}} t\right)-C_{2} \sin \left(n+\frac{K_{i j}}{M_{i}} t\right)\right]=\frac{\partial^{2}}{\partial n^{2}} \Delta \delta_{i}(n, t) .
$$

For the spatial domain derivation, consider a bus-topology consisting of $N$ nodes/buses. The Swing Equation describes the frequency dynamics of each node. Consider a disturbance at node $n=1$. The difference in frequency dynamics between node 1 and $N$ is as follows:

$$
M_{1} \dot{\omega}_{1}-M_{N} \dot{\omega}_{N}=P_{1}^{(m)}-P_{N}^{(m)}+\sum_{n=2}^{N} \frac{\vee_{n-1} \vee_{n}}{X_{n-1, n}} \sin \left(\delta_{n-1}-\delta_{n}\right)-P_{\zeta, 1}
$$

where $P_{\zeta, 1}$ denotes an imbalance disturbance at node $n=1$. For analytical simplicity, the following assumptions and approximations are relevant:

- $\quad$ Assume homogeneous edge parameters, $K_{n}=\frac{\vee_{n-1} \vee_{n}}{X_{n-1, n}}$,

- $\quad E\left\{P_{1}^{(m)}\right\} \approx E\left\{P_{N}^{(m)}\right\}$, thus $P_{1}^{(m)}-P_{N}^{(m)} \approx 0$,

- $\sin \left(\delta_{n-1}-\delta_{n}\right) \approx \Delta \delta_{n}(n)$,

- $\quad \frac{\partial^{2}}{\partial t^{2}} \Delta \delta_{n}(n)=\dot{\omega}_{n}-\dot{\omega}_{1}$,

- $M_{n}=M ; \forall n$.

Thus, (A13) becomes:

$$
\frac{\partial^{2}}{\partial t^{2}} \Delta \delta_{n}(n)+\frac{K_{n}}{M_{n}} \frac{\partial}{\partial t} \Delta \delta_{n}(n)=\frac{P_{\zeta, 1}}{M_{n}} .
$$

Like (A7), a second-order nonhomogeneous differential equation is obtained. The roots of the homogeneous part are complex, and the solution of this part is $P_{\zeta, 1} / K_{n}$. This means the solution is:

$$
\Delta \delta_{i}(n, t)=C_{1} \cos \left(n+\frac{K_{n}}{M_{n}} t\right)+C_{2} \sin \left(n+\frac{K_{n}}{M_{n}} t\right)-\frac{P_{\zeta, 1}}{K_{n}} .
$$

The second time derivative gives the RoCoF as follows:

$$
\frac{\partial^{2}}{\partial t^{2}} \Delta \delta_{i}(n, t)=\dot{\omega}_{n}
$$




$$
\dot{\omega}_{n}=-C_{1} \cos \left(n+\frac{K_{n}}{M_{n}} t\right)-C_{2} \sin \left(n+\frac{K_{n}}{M_{n}} t\right) .
$$

Thus, (A16) does indeed satisfy (A12). The solution meets the criteria for the wave equation, and the propagation speed of the RoCoF wave is:

$$
v=\sqrt{\frac{K}{M}}
$$

\section{References}

1. International Renewable Energy Agency (IRENA), International Energy Agency (IEA), Renewable Energy Integration in Power Grids (Technology Brief), 2015. Available online: http:/ /www.irena.org/publications/2015/Oct/Renewable-energy-integrationin-power-grids (accessed on 18 October 2018).

2. Kundur, P. Power System System Stability and Control, 1st ed.; Balu, N.J., Lauby, M.G., Eds.; McGraw-Hill: New York, NY, USA. 1994.

3. Fitzgerald, A.E.; Kingsley, C.; Umans, S.D. Electric Machinery, 6th ed.; McGraw-Hill: New York, NY, UAS, 2009.

4. Tielens, P.; Hertem, D.V. The relevance of inertia in power systems. Renew. Sustain. Energy Rev. 2016, 55, 999-1009. [CrossRef]

5. European Commission. Renewable Energy Progress Report, Report from the Commision to the European Parliament, the Council, the European Economic and Social committee and the Committee of the Regions. Available online: https://ec.europa.eu/ transparency/regdoc/rep/1/2017/EN/COM-2017-57-F1-EN-MAIN-PART-1.PDF (accessed on 18 October 2019).

6. F. Svenska Kraftnat Statnett and Energinet.dk. Challenges and Opportunities for the Nordic Power System, Technical Report, 2016. Available online: https:/ / energinet.dk/- / media/5D7F1D070E354130AD46183BF82D4FDB.pdf?la=da\&hash=009E60D2 7CD0DE20B2AC4AFDF6FB10A0E9187344 (accessed on 7 April 2017).

7. Coletta, T.; Bamieh, B.; Jacquod, P. Transient Performance of Electric Power Networks under Colored Noise. In Proceedings of the 57th IEEE Conference on Decision and Control, Miami, FL, USA, 17-19 December 2018; pp. 6163-6167.

8. Ulbig, A.; Borsche, T.S.; Andersson, G. Impact of Low Rotational Inertia on Power System Stability and Operation. IFAC Proc. Vol. 2014, 47, 7290-7297, doi:10.3182/20140824-6-ZA-1003.02615.

9. Guo, Y.; Summers, T.H. A Performance and Stability Analysis of Low-inertia Power Grids with Stochastic System Inertia. In Proceedings of the 2019 American Control Conference (ACC), Philadelphia, PA, USA, 10-12 July 2019; pp. 1965-1970. [CrossRef]

10. Dreidy, M.; Mokhlis, H.; Mekhilef, S. Inertia response and frequency control techniques for renewable energy sources: A review. Renew. Sustain. Energy Rev. 2017, 69, 144-155. [CrossRef]

11. Daz-Gonzlez, F.; Hau, M.; Sumper, A.; Gomis-Bellmunt, O. Participation of wind power plants in system frequency control: Review of grid code requirements and control methods. Renew. Sustain. Energy Rev. 2014, 34, 551-564. [CrossRef]

12. Morren, J.; Pierik, J.; de Haan, S.W.H. Inertial response of variable speed wind turbines. Electr. Power Syst. Res. 2006, 76, 980-987. [CrossRef]

13. Fang, J.; Li, H.; Tang, Y.; Blaabjerg, F. Distributed Power System Virtual Inertia Implemented by Grid-Connected Power Converters. IEEE Trans. Power Electron. 2018, 33, 8488-8499. [CrossRef]

14. Task force with members from REE, Terna, TransnetBW, 50Hertz Transmission, RTE, Swissgrid and Energinet.dk. Frequency Stability Evaluation Criteria for the Synchronous Zone of Continental Europe. European Network of Transmission System Operators for Electricity (Entsoe), 2016. Available online: https://docstore.entsoe.eu/Documents/SOC\%20documents/RGCE_ SPD_frequency_stability_criteria_v10.pdf (accessed on 11 June 2019).

15. Tasdighi, M.; Kezunovic, M. Automated Review of Distance Relay Settings Adequacy After the Network Topology Changes. IEEE Trans. Power Deliv. 2016, 31, 1873-1881. [CrossRef]

16. Tasdighi, M.; Kezunovic, M. Preventing transmission distance relays maloperation under unintended bulk DG tripping using SVM-based approach. Electr. Power Syst. Res. 2017, 142, 258-267. ISSN 0378-7796. [CrossRef]

17. Tyloo, M.; Pagnier, L.; Jacquod, P. The Key Player Problem in Complex Oscillator Networks and Electric Power Grids: Resistance Centralities Identify Local Vulnerabilities. Sci. Adv. 2019, 5, eaaw8359. [CrossRef]

18. Pirani, M.; Simpson-Porco, J.W.; Fidan, B. System-theoretic performance metrics for low-inertia stability of power networks. In Proceedings of the 2017 IEEE 56th Annual Conference on Decision and Control (CDC), Melbourne, VIC, Australia, 12-15 December 2017; pp. 5106-5111. [CrossRef]

19. Markovic, U.; Stanojev, O.; Aristidou, P.; Vrettos, E.; Callaway, D.S.; Hug, G. Understanding Small-Signal Stability of Low-Inertia Systems. Trans. Power Syst. 2021. [CrossRef]

20. Bhela, S.; Deka, D.; Nagarajan, H.; Kekatos, V. Designing Power Grid Topologies for Minimizing Network Disturbances: An Exact MILP Formulation. In Proceedings of the 2019 American Control Conference (ACC), Philadelphia, PA, USA, 10-12 July 2019; pp. 1949-1956. [CrossRef]

21. Tegling, E.; Bamieh, B.; Gayme, D.F. The Price of Synchrony: Evaluating the Resistive Losses in Synchronizing Power Networks. IEEE Trans. Control. Netw. Syst. 2015, 2, 254-266. 10.1109/TCNS.2015.2399193. [CrossRef] 
22. Murić, G.; Jorswieck, E.; Scheunert, C. Using LTI Dynamics to Identify the Influential Nodes in a Network. PLoS ONE 2016, 11, e0168514. [CrossRef]

23. Tyloo, M.; Coletta, T.; Jacquod, P. Robustness of Synchrony in Complex Networks and Generalized Kirchhoff Indices. Phys. Rev. Lett. 2018, 120, 084101. [CrossRef] [PubMed]

24. Thorp, J.S.; Seyler, C.E.; Phadke, A.G. Electromechanical wave propagation in large electric power systems. IEEE Trans. Circuits Syst. I Fundam. Theory Appl. 1998, 45, 614-622. [CrossRef]

25. Pagnier, L.; Jacquod, P. Inertia Location and Slow Network Modes Determine Disturbance Propagation in Large Scale Power Grids. PLoS ONE 2019, 14, e0213550. [CrossRef] [PubMed]

26. Ronellenfitsch, H.; Dunkel, J.; Wilczek, M. Optimal Noise-Canceling Networks. Phys. Rev. Lett. 2018. 121.208301 [CrossRef] [PubMed]

27. Parashar, M.; Thorp, J.S.; Seyler, C.E. Continuum modeling of electromechanical dynamics in large-scale power systems. IEEE Trans. Circuits Syst. I Regul. Pap. 2004, 51, 1848-1858. [CrossRef]

28. University Physics I Mechanics, Sound, Oscillations, \& Waves. Chapter 16.5: Energy and Power of a Wave, OpenStax University Physics. 2020, pp. 798-801. Available online: https://phys.libretexts.org/@go/page/4072 (accessed on 26 August 2020).

29. Power of a Wave. 2020. Available online: brilliant.org/wiki/power-of-a-wave/ (accessed on 23 May 2020).

30. Gea-Banacloche, J. University Physics I-Classical Mechanics. Chapter 12: Waves in One Dimension, University of Arkansas, LibreTexts, 2020, pp. 233-252. Available online: https://phys.libretexts.org/@go/page/22274 (accessed on 26 August 2020).

31. Pokapū Akoranga Pūtaiao. Waves as Energy Transfer. 2019. Available online: www.sciencelearn.org.nz/resources/120-waves-asenergy-transfer (accessed on 23 May 2020).

32. Hellmann, F.; Schultz, P.; Jaros, P.; Levchenko, R.; Kapitaniak, T.; Kurths, J.; Maistrenko, Y. Network-induced multistability through lossy coupling and exotic solitary states. Nat. Commun. 2020, 11, 592. [CrossRef]

33. Semlyen, A. Analysis of Disturbance Propagation in Power Systems Based on a Homogeneoue Dynamic Model. IEEE Trans. Power Appar. Syst. 1974, PAS-93, 676-684. [CrossRef]

34. Xu, Y.; Wen, F.; Ledwich, G.; Xue, Y. Electromechanical wave in power systems: theory and applications. J. Mod. Power Syst. Clean Energy 2014, 2, 163-172. 10.1007/s40565-014-0054-2. [CrossRef]

35. Farmer, W.J.; Rix, A.J. Optimising Power System Frequency Stability Using Virtual Inertia from Inverter-based Renewable Energy Generation. IET Renew. Power Gener. 2020. [CrossRef]

36. Wolter, J.; Lünsmann, B.; Zhang, X.; Schrder, M.; Timme, M. Quantifying transient spreading dynamics on networks. Chaos 2018, 28. [CrossRef] [PubMed]

37. Klein, D. Resistance-distance sum rules. Croatia Chem. Acta 2002, 73, 2002.

38. Xiao, W.; Gutman, I. Resistance distance and laplacian spectrum. Theor. Accounts 2003, 110, 284-289. [CrossRef]

39. Barmpoutis, D.; Murray, R.M. Networks with the Smallest Average Distance and the Largest Average Clustering. arXiv 2010, arXiv:1007.4031.

40. Poolla, B.K.; Bolognani, S.; Dörfler, F. Optimal placement of virtual inertia in power grids. IEEE Trans. Autom. Control 2017, 62, 6209-6220. [CrossRef]

41. Groß, D.; Bolognani, S.; Poolla, B.K.; Dörfler, F. Increasing the resilience of low-inertia power systems by virtual inertia and damping. Bulk Power Systems Dynamics and Control Symposium (IREP) 2017. Available online: http://people.ee.ethz.ch/ $\sim$ floriand/docs / Articles/Gross_IREP_2017.pdf (accessed on 15 May 2018).

42. Poolla, B.K.; Groß, D.; Dörfler, F. Placement and Implementation of Grid-Forming and Grid-Following Virtual Inertia and Fast Frequency Response. IEEE Trans. Power Syst. 2019, 34, 3035-3046. [CrossRef] 